\title{
Linear and nonlinear stability of a quasigeostrophic mixing layer subject to a uniform background shear
}

\author{
Luca Biancofiore $^{1}$ and Orkan M. Umurhan ${ }^{2,3}$ \\ ${ }^{1}$ Department of Mechanical Engineering, Bilkent University, Bilkent, 06800 Ankara, Turkey \\ ${ }^{2}$ Space Sciences Division, Ames Research Center, NASA, Moffett Field, California 94035, USA \\ ${ }^{3}$ SETI Institute, Mountain View, California 94043, USA
}

(Received 6 September 2018; published 30 January 2019)

\begin{abstract}
The aim of this work is to shed light by revisiting, from the kernel-wave (KW) perspective, the breakdown of a quasigeostrophic (QG) mixing layer (or vortex strip or filament) in atmosphere under the influence of a background shear. The QG mixing layer is modeled with a family of quasi-Rayleigh velocity profiles in which the potential vorticity $(\mathrm{PV})$ is constant in patches. From the KW perspective, a counterpropagating Rossby wave (CRW) is created at each PV edge, i.e., the edge where a PV jump is located. The important parameters of our study are (i) the vorticity of the uniform shear $m$ and (ii) the Rossby deformation radius $L_{d}$, which indicates how far the pressure perturbations can vertically propagate. While an adverse shear $(m<0)$ stabilizes the system, a favorable shear $(m>0)$ strengthens the instability. This is due to how the background shear affects the two uncoupled CRWs by shifting the optimal phase difference towards large (small) wave number when $m<0(m>0)$. As a finite $L_{d}$ is introduced, a general weakening of the instability is noticed, particularly for $m>0$. This is mainly due to the reduced interaction between the two CRWs when $L_{d}$ is finite. Furthermore, nonlinear pseudospectral simulations in the nominally infinite-Reynolds-number limit were conducted using as the initial base flow the same quasi-Rayleigh profiles analyzed in the linear analysis. The growth of the mixing layer is obstructed by introducing a background shear, especially if adverse, since the vortex pairing, which is the main growth mechanism in mixing layers, is strongly hindered. Interestingly, the most energetic configuration is for $m=0$, which differs from the linear analyses for which the largest growth rates were found for a positive $m$. In the absence of a background shear additional modes are subharmonically triggered by the initial disturbance enhancing the turbulent character of the flow. We also confirm energy spectrum trends for broken-down mixing layers reported in the literature. We interpret the character of mixing-layer breakdown as being a phenomenological hybrid of Kraichnan's [R. H. Kraichnan, Phys. Fluids 10, 1417 (1967)] direct enstrophy cascade picture and the picture of self-similar vortex production.
\end{abstract}

DOI: 10.1103/PhysRevFluids.4.013803

\section{INTRODUCTION}

Idealized two-dimensional (2D) flow and turbulence continue to serve as an indispensable platform to study the physical properties of various fluid dynamical processes and effects. Two dimensionality arises naturally in systems exhibiting dynamically restricted dimensions like in planetary atmospheric flows and soap bubbles. Despite often cited criticisms implicating their lack of real-world relevance, the study of idealized flows like 2D Navier-Stokes equations offers a challenging yet intellectually traversable setting to examine complex physical notions, for example, the recently uncovered conformal invariance between the inverse cascade of $2 \mathrm{D}$ turbulence $[1,2]$ and critical phenomena of 2D statistical mechanics [3,4]. 


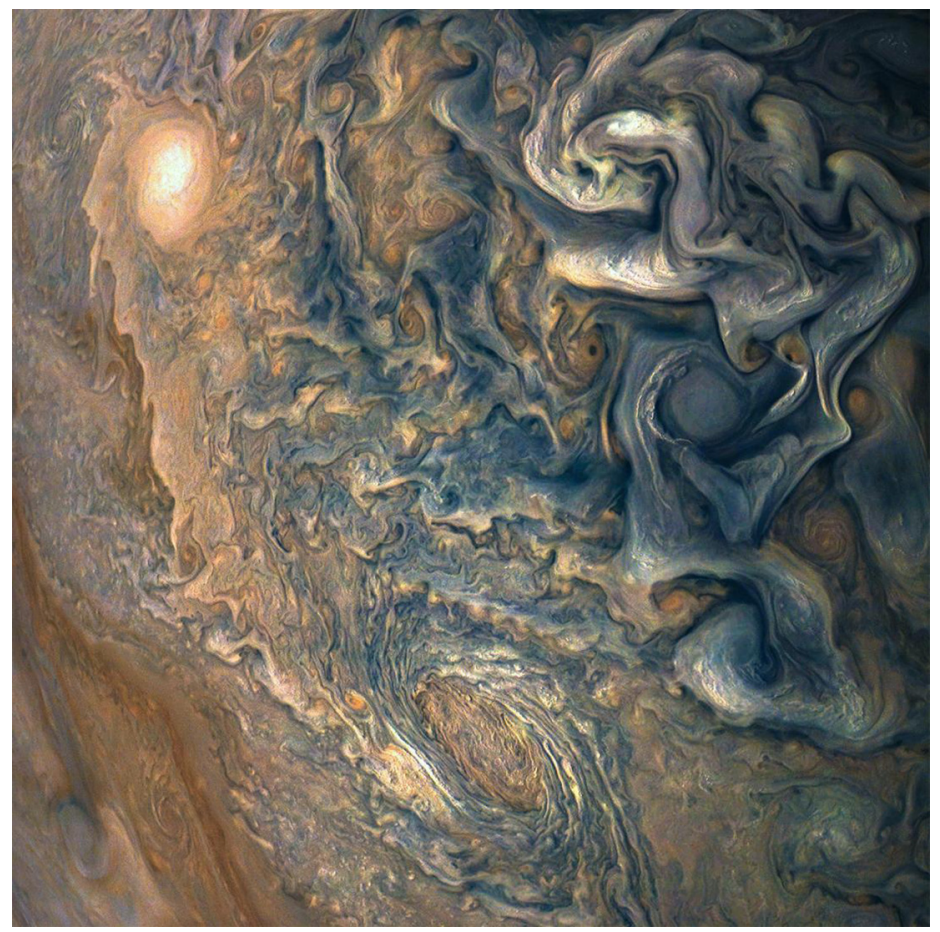

FIG. 1. Juno image of Jupiter's cloud tops near northern latitude $48.9^{\circ}$. The horizontal scale of the image is approximately $95000 \mathrm{~km}(9.3 \mathrm{~km} /$ pixel). Being in the quasigeostrophic regime, these regions exhibit filamentary structure as well as large-scale coherent vortices. The Rossby deformation radius at these latitudes is approximately $20000 \mathrm{~km}$. (Image credit: NASA, JPL-Caltech, SwRI, MSSS, Gerald Eichstadt, and Sean Doran.)

Of interest to this study is the dynamical nature of the transition of vorticity strips in 2D flows. Sometimes called the mixing layer, such parallel strips are idealized as streamwise oriented patches of two different constant vorticities, one value inside and another outside. The mixing layer is considered a canonical test bed to study the breakdown of small-scale filaments due to its own vorticity induced velocities with or without the influence of an externally imposed shear [5]. This has applications in understanding the dynamics in the forward cascade inertial range of 2D turbulence studies. The simplest mixing layer is the so-called Rayleigh layer [6-8] in which the outside vorticity is zero, which leads to a configuration in which the streamwise velocities on the outside are two different constants and the region within the strip has a streamwise velocity that linearly connects to the outer regions. The Rayleigh layer is unstable, resulting in its roll-up into smaller coherent vortices with attendant interwoven fine-scale filamentary structures. This basic mixing-layer setting, as a fundamental qualitative physical model, is encountered frequently in terrestrial and planetary atmospheric flows, e.g., as recent Juno mission images of cloud top formations in Jupiter's upper atmosphere clearly shows (see Fig. 1).

We are partial to the counterpropagating Rossby wave (CRW) perspective $[9,10]$ in understanding the breakdown of the mixing layer $[11,12]$. In this picture, whose generality was first recognized and systematically developed by Baines and Mitsudera [13], each perturbed edge of the idealized mixing layer supports a Rossby edge wave which azimuthally propagates against the local flow. Each Rossby edge wave instantly induces a far-field velocity (action at a distance) so that the two edge waves can constructively interact with one another if the conditions are right: If the sum of the intrinsic Rossby edge-wave speed and the local flow velocity are nearly equal for both edge waves, then the system can enter a resonance due to the action-at-a-distance effect resulting 

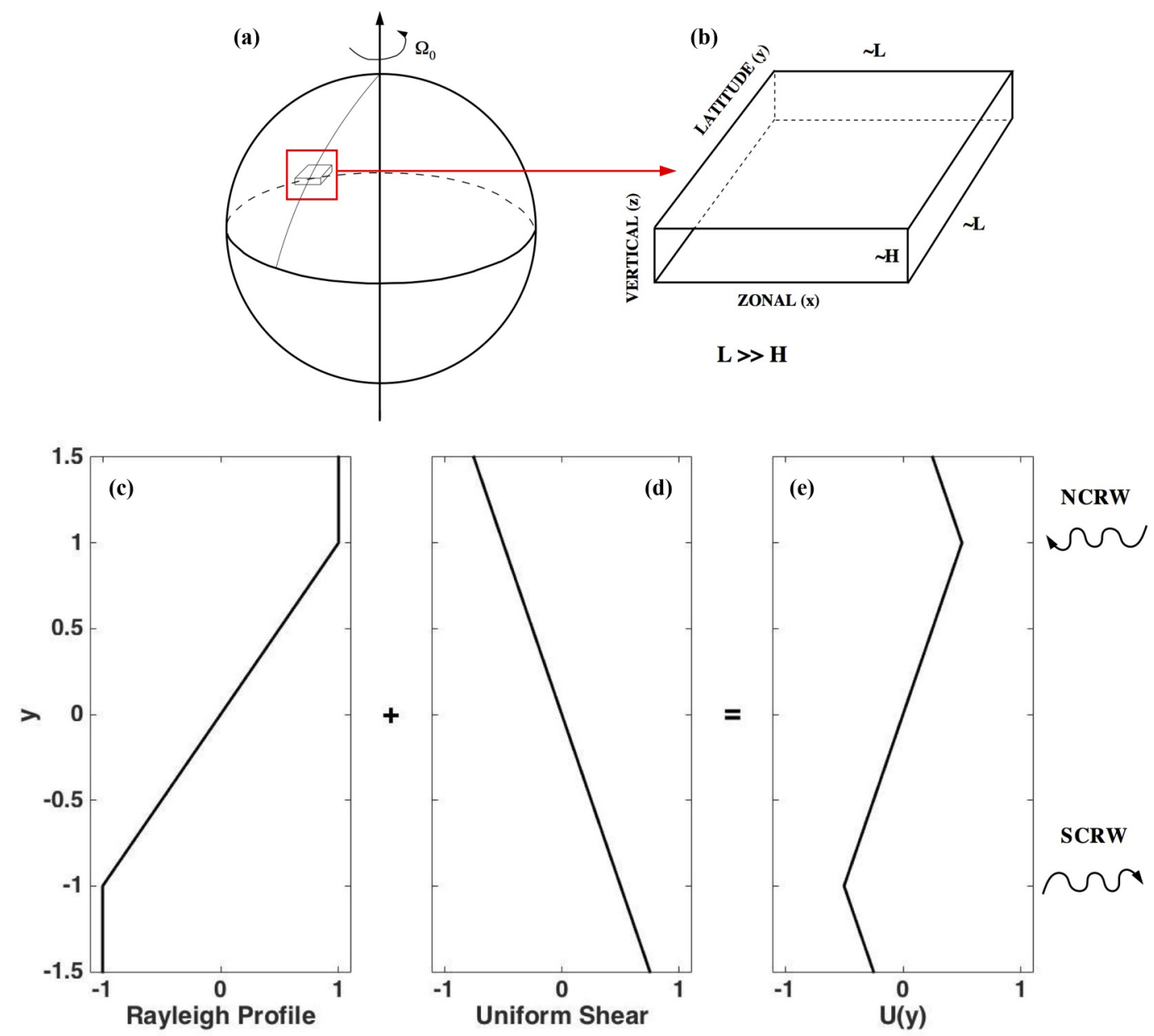

FIG. 2. From (a) the planetary scale in spherical coordinates to the synoptic scale in Cartesian coordinate. (e) Velocity profile for $L_{d}=\infty$, i.e., (c) the Rayleigh profile plus (d) the uniform shear. Two CRWs are formed at the two vorticity edges: NCRW at the northern edge and SCRW at the southern edge.

in mutual amplitude growth. As a general mechanism, sometimes referred to as the kernel-wave (KW) perspective [14], this can apply to any two pairs of localized edge waves of a mixing layer irrespective of the fundamental disturbance type, be it gravity waves, magnetohydrodynamic waves, capillary waves, or others [14-19]. Indeed, this conceptual framework has been usefully applied toward the interpretation of various geophysical flow phenomena, e.g., Holmboe instability [20], baroclinic instability [9], the Eady problem [21], the Charney problem [22], and the polar polygons recently observed on Saturn [23].

In the context of quasigeostrophic (QG) flows, typifying midlatitude to high-latitude synopticscale dynamics in atmospheres and oceans [24] [also see the sketch in Fig. 2(a)], the fate of mixing layers depends sensitively on the Rossby radius of deformation [25] $L_{d}$, which is approximately $1000 \mathrm{~km}$ in the Earth's midlatitude atmosphere and defines the synoptic scale. On Jupiter and Saturn this figure is 15-35 times larger [26]. A cursory glance at the operator relating the stream function to the potential vorticity (PV) [25] readily indicates that $L_{d}$ acts to diminish the ability of a local patch of PV to induce far-field velocity fluctuations: Large values of $L_{d}$ means that the stratification is strong and consequently the dynamical reach of a deformed edge wave is far out (and limits the classical 2D case as $L_{d} \rightarrow \infty$ ), while when $L_{d}$ is small the dynamical influence of the same edge 
wave is muted by an exponential factor $\sim \exp \left(-r / L_{d}\right)$, where $r$ is the distance from the edge wave to the point of influence.

Another factor characterizing a mixing layer's stability is the sense of any applied background constant-shear flow profile. To give an example, a uniform shear strongly affects the stability of filaments created in the vicinity of intense rotating coherent vortices. The filaments are rapidly aligned with the circulating flow due to the differential rotation and then shear is the prime factor affecting stability. If adverse, constant shear acts to suppress instability [5]. From the viewpoint of the KW perspective, one might expect this to be so because the resonance criterion between the two opposing edge waves is hindered as the mean flow at the respective edges is unable to compensate for the intrinsic Rossby wave speed of the respective disturbed edges. On its own right, examining the fate of a QG mixing layer subject to an external constant shear offers physical insight into the workings of the forward enstrophy cascade in a fully turbulent 2D setting. In particular, it sheds light upon the influence of (spectrally) nonlocal interactions, here being the forcing brought down from the global shear upon small-scale filaments. In this way we see the KW perspective as offering another helpful tool in understanding the characteristic development of 2D turbulence.

In this study we revisit the problem of the breakdown of mixing layers subject to an external constant shear in the QG model setting (Sec. II). Our interest is to interpret the results found by Dritschel and Waugh [5,25] within the KW framework. In particular, we consider the conditions leading to the mixing-layer breakdown and rationalize these linear stability conditions in terms of the ability (or lack thereof) of individual edge waves to achieve frequency resonance as a function of the background imposed constant shear and $L_{d}$ (Sec. III). We further test the robustness of the KW perspective by conducting a series of fully nonlinear simulations of the breakdown of QG mixing layers (Sec. IV). In particular, we recast the original setup of Dritschel and Waugh instead as a composite flow made up of a so-called quasi-Rayleigh profile plus an externally imposed steady shear and making sure, according to the KW perspective, that the velocities and jumps in PV are equivalent to the setup examined by Waugh and Dritschel [25] (Sec. IV A). We devote the rest of Sec. IV to assessing the nonlinear development of the breakdown of the mixing layer in which we recover the $k^{-11 / 3}$ spectral energy distribution previously shown to hold in the enstrophy cascade regime of $L_{d}=\infty$ mixing layers [27,28]. Furthermore, we examine the spectral energy slope in the enstrophy cascade regime as a function of $L_{d}$, finding that its slope $\left(k^{-\delta}, \delta\right.$ is the slope) indeed steepens $(5 / 3<\delta<11 / 3)$ as $L_{d}$ approaches 1 . We also characterize the shearwise spread of the disrupted mixing layer as a function of amplitude and sense of the applied background constant shear. Finally, through the lens of the KW perspective, we tentatively offer an interpretation to explain the statistical quality of the broken-down shear layer as being phenomenologically indicative of a process that lies between the Kraichnan or Gilbert [1,27] explanation of enstrophy cascade in forced-dissipative 2D turbulence and the picture of the self-similar production of coherent vortices down to the dissipative scales [29-32]. Section V summarizes our results.

\section{MODEL AND GOVERNING EQUATIONS}

In this section we first present the model analyzed in this paper (Sec. II A). Afterward, in Sec. II B we use the KW perspective to arrive at a dynamical system able to describe the linear stability of this model.

\section{A. Model}

We consider the planetary atmosphere to be a thin fluid layer lying atop a spherical surface rotating with an angular velocity $\Omega_{0}$ [see Fig. 2(a)]. If we assume that the planetary atmosphere is a fast rotator we can consider only a Cartesian section [Fig. 2(b)], where $x$ is the zonal (eastward) direction, $y$ is the latitudinal (poleward) direction, and $z$ is the vertical (altitude) direction. Let $\mathcal{U}$ be the horizontal velocity scale which occurs on the horizontal length scale $L$ and let $H$ denote the vertical scale, i.e., the pressure scale height of the atmosphere. If we assume infinitely fast 
hydrostatic adjustment and work in an $f$ plane (i.e., no variations due to the Coriolis effect are considered in all the motions), the potential vorticity and its evolution in a two-dimensional incompressible quasigeostrophic flow is given by

$$
q=\nabla^{2} \psi-\frac{1}{L_{d}^{2}} \psi, \quad \frac{d q}{d t}=0,
$$

where $\psi$ is the stream function given by $u=-\frac{\partial \psi}{\partial y}$ and $v=\frac{\partial \psi}{\partial x}$, with $u$ and $v$ the zonal and latitudinal components of the velocity, respectively, and $L_{d}$ is the Rossby radius of deformation. The Rossby radius of deformation is a measure of how far the pressure perturbations can travel at the flow timescale and is given by $L_{d}^{2}=\frac{H^{2} N^{2}}{4 \Omega_{0}^{2}}$, where $N^{2}$ is the Brunt-Väisälä frequency, i.e., the frequency of the buoyancy vertical oscillations. For further details on the physically motivated derivation of Eq. (1), the reader is referred to Ref. [8].

Our model is represented by the family of the nondimensional velocity profiles already analyzed by Waugh and Dritschel [25],

$$
U(y)= \begin{cases}L_{d}\left[e^{-y / L_{d}} \sinh \left(\frac{1}{L_{d}}\right)+m e^{-1 / L_{d}} \sinh \left(\frac{y}{L_{d}}\right)\right] & \text { for } y>1 \\ L_{d}(m+1) e^{-1 / L_{d}} \sinh \left(\frac{y}{L_{d}}\right) & \text { for }|y|<1 \\ L_{d}\left[-e^{y / L_{d}} \sinh \left(\frac{1}{L_{d}}\right)+m e^{-1 / L_{d}} \sinh \left(\frac{y}{L_{d}}\right)\right] & \text { for } y<-1\end{cases}
$$

that has piecewise constant potential vorticity

$$
Q(y)= \begin{cases}-m e^{(D-2) / 2 L_{d}} & \text { for }|y|>1 \\ -\left(m e^{(D-2) / 2 L_{d}}+1\right) & \text { for }|y|<1,\end{cases}
$$

where $D$ is a distance much greater than both the strip width (equal to 2) and the radius of deformation $L_{d}$. The limit for $L_{d} \rightarrow \infty$ of the family velocity profile depicted by Eq. (2) [illustrated in Fig. 2(e)] is the Rayleigh model [6,33] [Fig. 2(c)] sheared by a uniform shear $m$ [Fig. 2(d)],

$$
U(y)= \begin{cases}m y+1 & \text { for } y>1 \\ (m+1) y & \text { for }|y|<1 \\ m y-1 & \text { for } y<-1\end{cases}
$$

\section{B. Kernel-wave perspective}

The velocity $\mathbf{v}$ and the potential vorticity $q$ can be linearized with respect to the basic state, $\mathbf{v}=\left(U+u^{\prime}, v^{\prime}\right)$ and $q=Q+q^{\prime}$, where the capital letters and the primes indicate the basic state [Eqs. (2) and (3)] and perturbation, respectively. The linearized potential vorticity equation $\frac{D q}{D t}=0$ gives

$$
\left[\frac{\partial}{\partial t}+U(y) \frac{\partial}{\partial x}\right] q^{\prime}=-v^{\prime} \frac{d Q(y)}{d y}=-v^{\prime}[\delta(y-1)-\delta(y+1)],
$$

where $\delta$ symbolizes the Dirac delta function.

Applying the KW perspective [11], we look for a vorticity perturbation field that is concentrated on the discontinuities of the base flow vorticity, i.e., on the two edges of the velocity profile. Then, for a single Fourier component with wave number $k$ of the form $e^{i k x}$, Eq. (5) can be rewritten as

$$
\hat{q}^{\prime}=q_{S}(k, t) \delta(y+1)+q_{N}(k, t) \delta(y-1),
$$

where $q_{S}(k, t)$ and $q_{N}(k, t)$ represent vorticity waves, i.e., the CRWs, at southern and northern edge, respectively. From Eq. (1) it follows that $\hat{q}^{\prime}=-\mathcal{L} \hat{\psi}^{\prime}$, with $\mathcal{L}=d^{2} / d y^{2}-\bar{k}^{2}$, where $\bar{k}^{2}=k^{2}+\frac{1}{L_{d}^{2}}$. The Green's function of $-\mathcal{L}$ for an unbounded domain is

$$
G\left(y, y^{\prime}, \bar{k}\right)=-\frac{i}{2} \exp \left(-\bar{k}\left|y-y^{\prime}\right|\right)
$$


and the perturbation stream function is then obtained,

$$
\hat{\psi}^{\prime}=-\frac{1}{2 \bar{k}}\left[q_{S}(k, t) e^{-\bar{k}|y+1|}+q_{N}(k, t) e^{-\bar{k}|y-1|}\right],
$$

where $\psi^{\prime}$ satisfies $u^{\prime}=\frac{\partial \psi^{\prime}}{\partial y}$ and $v^{\prime}=-\frac{\partial \psi^{\prime}}{\partial x}$, i.e., in Fourier space $\hat{u}^{\prime}=\partial_{y} \hat{\psi}^{\prime}$ and $\hat{v}^{\prime}=i k \hat{\psi}^{\prime}$. Substituting Eqs. (6) and (8) into Eq. (5), one is left with

$$
\begin{aligned}
& \dot{q}_{S} \delta(y+1)+\dot{q}_{N} \delta(y-1)+i k U(y)\left[q_{S} \delta(y+1)+q_{N} \delta(y-1)\right] \\
& \quad=i \frac{k}{2 \bar{k}}\left[q_{S}(k, t) e^{-\bar{k}|y+1|}+q_{N}(k, t) e^{-\bar{k}|y-1|}\right][\delta(y-1)-\delta(y+1)],
\end{aligned}
$$

where the notation $\dot{q}_{S, N}$ denotes $\frac{d q_{S, N}}{d t}$.

Calculating Eq. (9) at the edges, i.e., $y=\mp 1$, the evolution of the vorticity perturbation is obtained,

$$
\dot{q}=M q
$$

where

$$
\boldsymbol{M}=i k\left[\begin{array}{cc}
-\frac{1}{2 \bar{k}}+L_{d}(m+1) e^{-1 / L_{d}} \sinh \frac{1}{L_{d}} & -\frac{e^{-2 \bar{k}}}{2 \bar{k}} \\
\frac{e^{-2 \bar{k}}}{2 \bar{k}} & \frac{1}{2 \bar{k}}-L_{d}(m+1) e^{-1 / L_{d}} \sinh \frac{1}{L_{d}}
\end{array}\right]
$$

The terms in the entry diagonal are the phase speed of the CRWs taken in isolation $c_{N, S}=$ $\pm\left[\frac{1}{2 k}-L_{d}(m+1) e^{-1 / L_{d}} \sinh \frac{1}{L_{d}}\right]$, while the off-diagonal terms represent the interaction coefficient $\gamma=\frac{k e^{-2 \bar{k}}}{2 \bar{k}}$. The eigenvalues of the matrix $M$,

$$
\lambda_{1,2}= \pm \frac{1}{2} \frac{k}{\bar{k}} \sqrt{e^{-4 \bar{k}}-\left[1-\bar{k} L_{d}\left(1-e^{-2 / L_{d}}\right)(m+1)\right]^{2}},
$$

indicate the temporal response of each of the system's normal modes. We note that the temporal response indicated by the eigenvalues in (11) is equivalent to that obtained by more traditional methods.

We note that in classical textbook treatments (e.g., Sec. 6.2.4 of Ref. [24]) the above system is a $4 \times 4$ matrix system. This apparent contradiction is easy to explain since our approach is based on the Heifetz et al. paper [11] in which they assume that the stream function is continuous at the two PV jumps. For this reason, in our model the matrix system is $2 \times 2$. By assuming a continuous stream function also in Vallis's treatment we can obtain, after some calculations, exactly the same stream function of Ref. [11].

\section{LINEAR STABILITY ANALYSIS}

In this section the linear stability of the dynamical system depicted by Eq. (11) is analyzed. In particular, we study the effect of (i) the background shear, in Sec. III A, and (ii) the Rossby deformation radius, in Sec. III B. Finally, in Sec. III C, a detailed analysis about the waves amplitude and their phases is conducted.

\section{A. Influence of the uniform background shear}

We illustrate in Fig. 3(a) the growth rate $\lambda_{r}$, i.e., the real part of the eigenvalues in Eq. (11), as a function of the wave number $k$ for different values of the uniform shear background $m$ while setting $L_{d}=\infty$. Clearly, an adverse shear $(m<0)$ decreases the maximum growth rate and reduces the range of unstable wave numbers. Moreover, this range is shifted towards small wavelengths. A positive value of $m$ conversely enhances the maximum growth rate while still decreasing the cutoff number. These results are in agreement with previous works on the effect of a background shear on the destabilization of a vorticity filament $[5,25]$. 

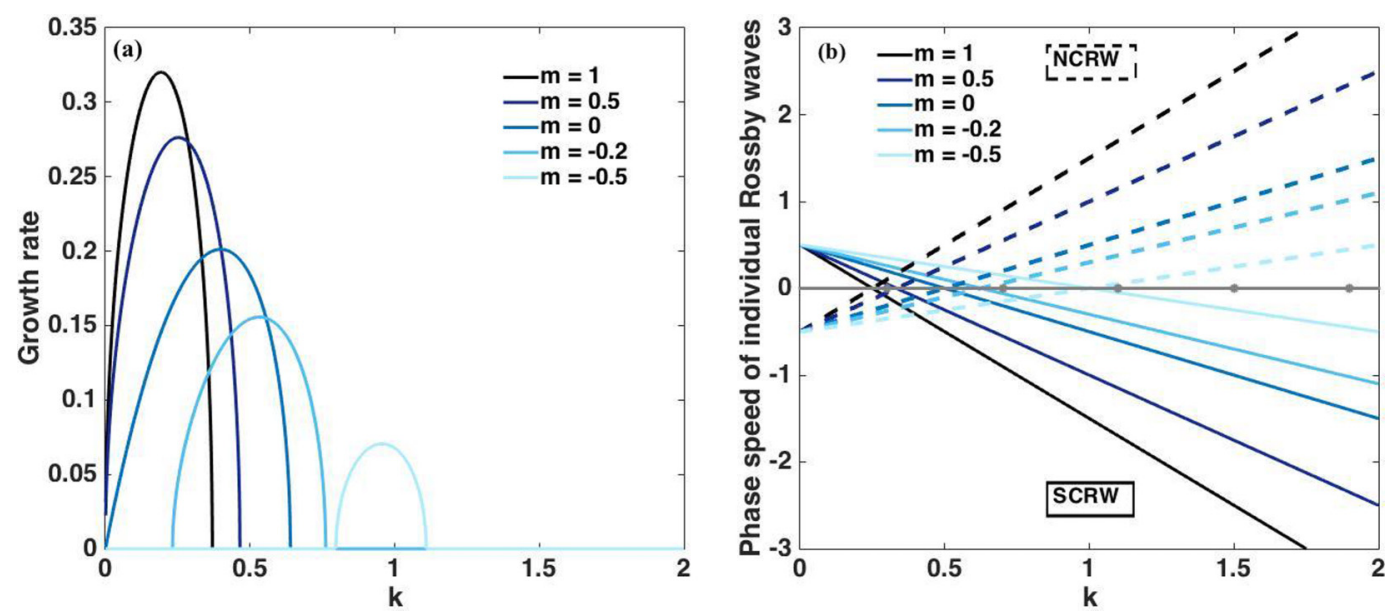

FIG. 3. (a) Growth rate vs wave number $k$ and (b) the phase speed of the two CRWs taken in isolation for different values of the uniform shear $m$ and $L_{d}=\infty$. In (b) dashed lines depict the NCRW, while solid lines depict the SCRW. A NCRW (SCRW) is counterpropagating if its phase speed is below (above) the gray line, which depicts the average base flow velocity $\left(U_{a v}=0\right)$.

In Fig. 3(b) the phase speeds of the two CRWs taken in isolation are illustrated for the same cases as in Fig. 3(a). Dashed lines depict the northern CRW (NCRW), while solid lines depict the southern CRW (SCRW). The range of unstable wave numbers encloses the wave number $k_{\text {equal }}$ in which the individual waves have the same phase speed $\left(c_{N}=c_{S}\right)$. This is consistent with the Hayashi-Young criterion [34], i.e., two waves must have a similar phase speed to be able to phase lock and so generate the instability. Note that the effect that each CRW has on the other's phase speed is not considered while calculating the phase speeds (see Sec. III C for more details). However, this effect cannot significantly modify the phase speed; for this reason, the range of unstable wave numbers surrounds $k_{\text {equal }}$. Furthermore, $k_{\text {equal }}$ is shifted towards smaller (large) wave numbers for positive (negative) values of $m$. The background shear modifies the phase speeds of the CRWs, allowing the interaction in a different range of wave numbers. This explains why the range of unstable wave numbers is strongly modified by the uniform shear.

\section{B. Influence of the Rossby deformation radius}

In this section a finite value of the Rossby deformation radius is introduced. The growth rates are illustrated in Fig. 4(a), where the background shear is set equal to $m=0$ and $L_{d}$ varies. The maximum growth rate is significantly damped with the introduction of a finite $L_{d}$, as has been previously noted. Furthermore, promotion of stabilization upon reduction of $L_{d}$ is not surprising since the dynamical influence of each edge wave becomes increasingly muted as stratification weakens in a later rotation. In particular, the ratio between kinetic energy and available potential energy is proportional to $L_{d}^{2}$ [24]. This means that if $L_{d}$ is infinite, the ability of a PV disturbance to induce a far-field horizontal velocity response is maximized, while less kinetic energy is available in the horizontal motions as $L_{d}$ decreases, clearly inducing a stabilization.

In Fig. 4(b) we show the phase speed of the two individual CRWs for the cases analyzed in Fig. 4(a). Dashed lines represent the NCRW, while solid lines represent the SCRW as in Fig. 3(b). Interestingly, a decrease in the Rossby radius of deformation damps the counterpropagating nature of the waves. Less or even no counterpropagation is a stabilizing symptom since counterpropagation helps the phase locking of the two waves [34,35], allowing their resonance and then generating the instability. As the deformation radius is finite, some of the wave energy associated with the Rossby wave of a given edge is redirected into vertical storage. This stored energy would otherwise have 

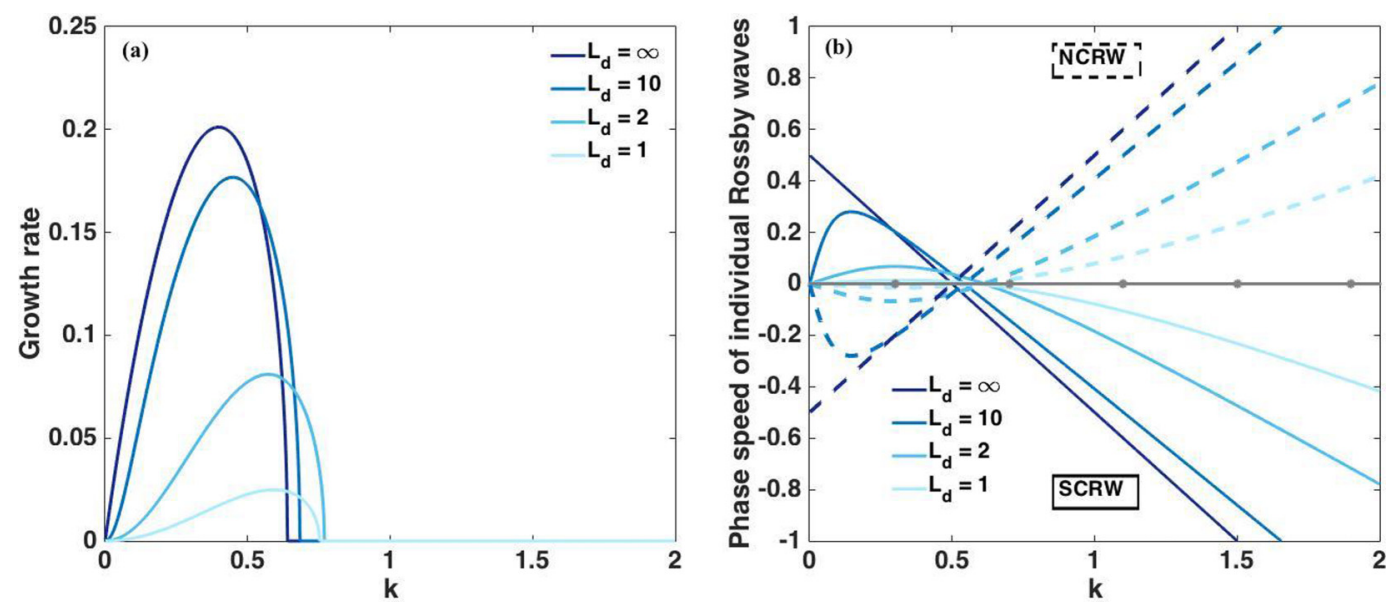

FIG. 4. (a) Growth rate vs wave number $k$ and (b) the phase speed of the two CRWs taken in isolation for $m=0$ and different values of $L_{d}$. In (b) dashed lines depict the NCRW, while solid lines depict the SCRW. A NCRW (SCRW) is counterpropagating if its phase speed is below (above) the gray line, which depicts the average base flow velocity $\left(U_{a v}=0\right)$.

been harnessed into inducing a velocity field at the opposite edge, which would have played a role in countering the base velocity of the opposite edge. For this reason, counterpropagation is thus restrained or, if the conditions are right, completely eliminated.

In Fig. 5 the contours of the growth rate are illustrated in the $k-m$ plane for $L_{d}=\infty$ [Fig. 5(a)], $L_{d}=10$ [Fig. 5(b)], $L_{d}=2$ [Fig. 5(c)], and $L_{d}=1$ [Fig. 5(d)]. This figure confirms the trend previously depicted: A negative $m$ and a decrease in the Rossby deformation radius stabilize the system. In particular, a finite value of $L_{d}$ is more efficient for positive values of $m$.

\section{Amplitudes and phases of the waves}

It is possible to write the two vorticity waves $q_{S}$ and $q_{N}$ in this manner

$$
\begin{gathered}
q_{S}=Q_{S}(k, t) e^{i \epsilon_{S}(k, t)}, \\
q_{N}=Q_{N}(k, t) e^{i \epsilon_{N}(k, t)},
\end{gathered}
$$

where $Q_{S}$ and $Q_{N}$ are the amplitudes of the southern and northern CRWs and $\epsilon_{S}$ and $\epsilon_{N}$ are their phases, respectively. In this manner we can arrive at the system

$$
\begin{aligned}
\dot{Q}_{S} & =\gamma Q_{S} \sin (\Delta \epsilon), \\
\dot{Q}_{N} & =\gamma Q_{N} \sin (\Delta \epsilon), \\
\dot{\epsilon}_{S} & =-k c_{S}-\gamma \frac{Q_{N}}{Q_{S}} \cos (\Delta \epsilon), \\
\dot{\epsilon}_{N} & =-k c_{N}+\gamma \frac{Q_{S}}{Q_{N}} \cos (\Delta \epsilon),
\end{aligned}
$$

where $\Delta \epsilon=\epsilon_{N}-\epsilon_{S}$ is the phase difference between the two CRWs. From Eqs. (13) we can understand that (i) the waves can grow just interacting with each other and (ii) the interaction strength depends on both $\gamma$ and $\Delta \epsilon$. When the phase difference is $0<\Delta \epsilon<\pi$ we are in the growing phase-locking configuration, while if $-\pi<\Delta \epsilon<0$ it is a decaying configuration [33].

We can obtain amplitudes and phases by computing the eigenvectors $V_{i}=\left[v_{i, S}, v_{i, N}\right]$ of the matrix $M$ [Eq. (11)], where the index $i$ depicts a different mode. In particular, the amplitude of 

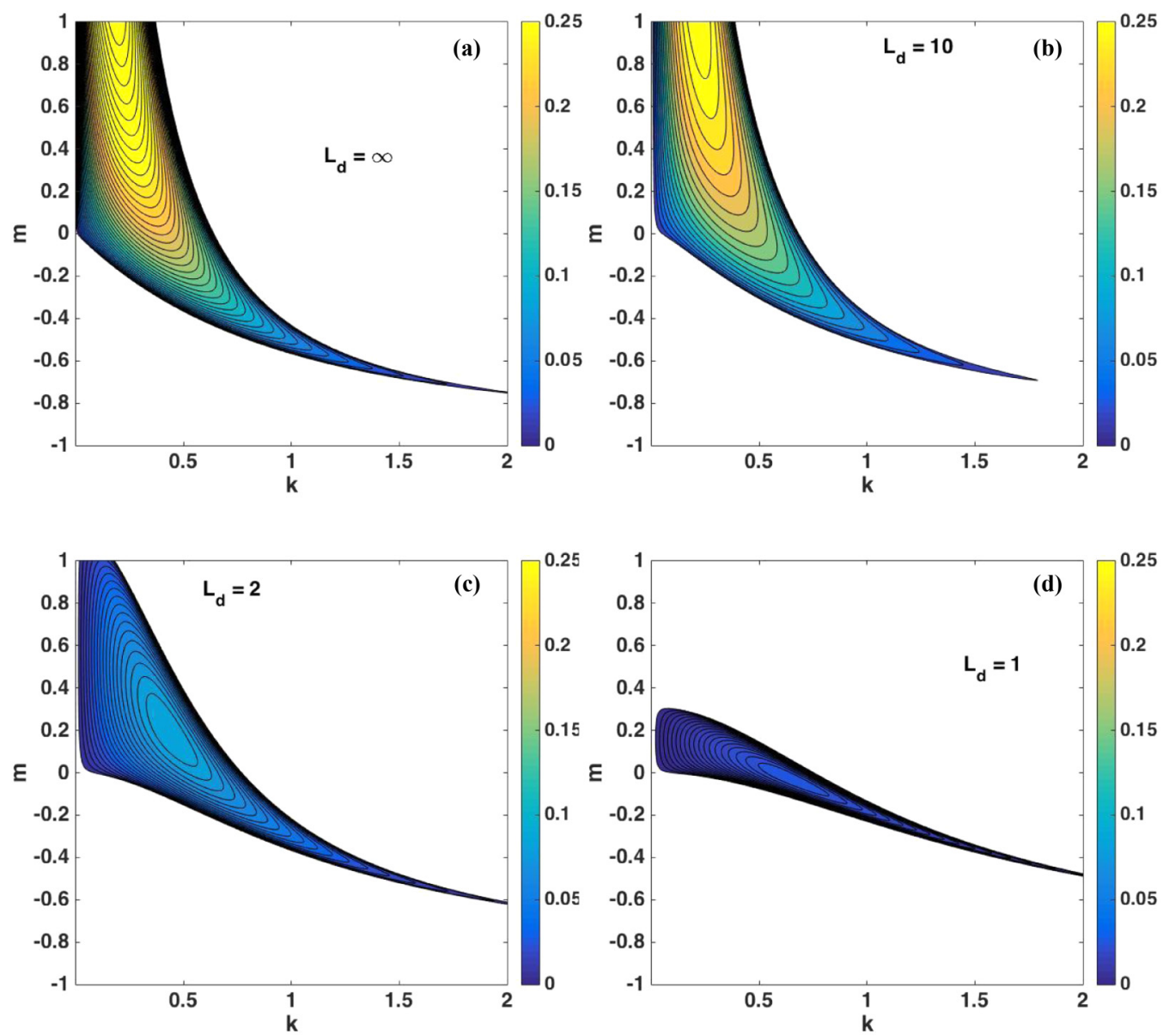

FIG. 5. Growth rate in the plane $k-m$ for (a) $L_{d}=\infty$, (b) $L_{d}=10$, (c) $L_{d}=2$, and (d) $L_{d}=1$.

SCRW is obtained with $Q_{S}=\left|v_{i, S}\right|$, where $v_{i, S}$ is the $S$ component of the eigenvector $V_{i}$, while its phase is $\epsilon_{S}=\arg \left(v_{i, S}\right)$. Similarly, $Q_{N}$ and $\epsilon_{N}$ can be obtained. Note that the choice of the index $i$ ( $i=1$ or 2 ) is not important to determine the amplitude and the phase of the waves.

In Fig. 6 we illustrate the phase difference $\frac{\Delta \epsilon}{\pi}$ and the interaction coefficient $\gamma$ for $L_{d}=\infty$ and different values of $m$. The phase difference corresponding to the maximum growth rate $\Delta \epsilon_{\mathrm{opt}}=$ $0.65 \pi$ for $m=0$ is highlighted by a dashed line. Then, for $m=0$ the gravest normal mode is not for $\Delta \epsilon=\frac{\pi}{2}$, where we have the maximal instantaneous growth rate as seen in Eqs. (13a) and (13b). To explain this we have to consider the two conditions to have phase locking [34]: The waves must have the same (i) amplitude $Q_{S}=Q_{N}$ and (ii) phase speed $\dot{\epsilon}_{1}=\dot{\epsilon}_{2}$. The latter conditions is not respected for $\Delta \epsilon=\frac{\pi}{2}$ since the waves cannot affect each other's phase speed [see Eqs. (13c) and (13d)] and then the interaction could occur just if $c_{S}=c_{N}$, which is not true. In particular, if $-\frac{\pi}{2}<\Delta \epsilon<\frac{\pi}{2}$ the two waves hinder their self-propagation against the mean flow; conversely, if $\frac{\pi}{2}<\Delta \epsilon<\frac{3}{2} \pi$ they help the counterpropagation $[19,33]$. Phase locking is favored when interaction hinders the CRW counterpropagation. The maximum modal growth rate occurs where there is a trade-off between the exponential increase of the interaction coefficient [see Fig. 6(b)] at small wave numbers and the need of a hindering configuration to enable phase locking. The reader is referred to Ref. [33] for more details on the CRW phase locking. 

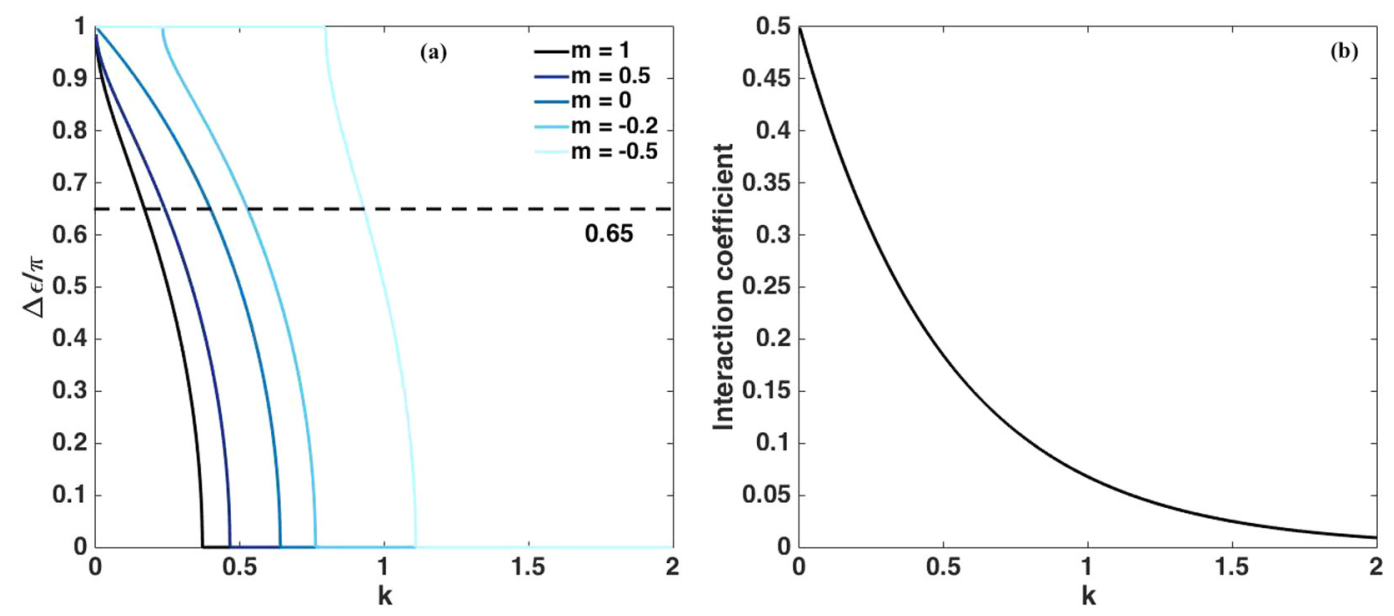

FIG. 6. (a) Phase difference $\frac{\Delta \epsilon}{\pi}$ and (b) interaction coefficient for $L_{d}=\infty$ and different values of $m$. In (a) the dashed line represents the phase difference corresponding to the maximum growth rate $\Delta \epsilon=0.65$ for $m=0$.

The interaction coefficient does not depend on the presence of the background shear, while the phase difference is modified by $m$ [see Fig. 6(a)]. The optimal phase difference $0.65 \pi$ is shifted towards long (short) wavelengths for positive (negative) $m$. This can explain why the instability is moved by a favorable (adverse) shear towards long (short) wavelengths as observed in Fig. 3(b). Since the interaction coefficient does not change with the introduction of the background shear, the optimal phase difference corresponds now to a stronger or weaker interaction, depending on the sign of $m$, as it is possible to see in Fig. 6(b). It should be noticed that the introduction of the background shear will modify the value of the optimal phase difference since the phase speed of the isolated CRWs depends on $m$ [see Fig. 3(b)]. However, we can assume that the dependence is not significant at least from a qualitative point of view. This point will be discussed later in more detail.
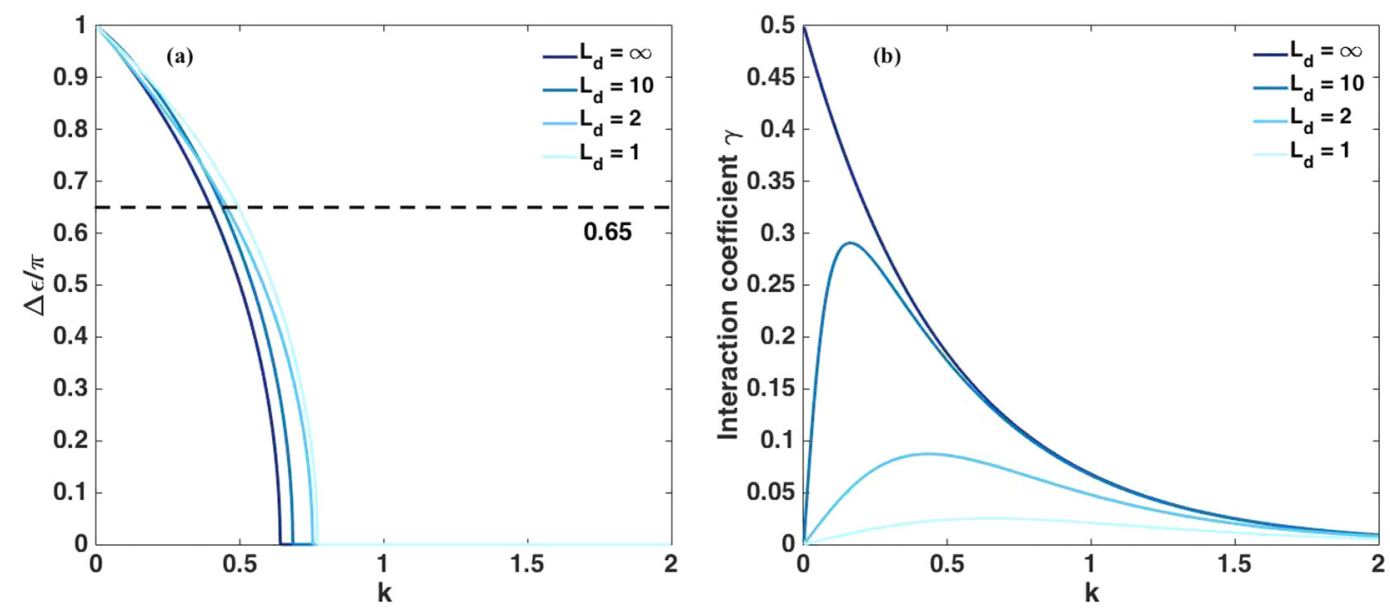

FIG. 7. (a) Phase difference $\frac{\Delta \epsilon}{\pi}$ and (b) the interaction coefficient $\gamma$ vs wave number for different values of $L_{d}$ and $m=0$. The dashed line shows the value of the optimal phase difference $0.65 \pi$ for the Rayleigh model (so $m=0$ and $L_{d}=\infty$ ) [33]. 
In Fig. 7 we show the phase difference $\frac{\Delta \epsilon}{\pi}[$ Fig. 7(a)] and the interaction coefficient $\gamma$ [Fig. 7(b)] for $m=0$ and different values of the Rossby deformation radius. The phase difference is not strongly modified by $L_{d}$, however the CRW interaction becomes weaker. This is in agreement with the behavior of the growth rate observed in Fig. 4(a): $L_{d}$ significantly damps the instability, but the range of unstable wave number is almost untouched.

In Fig. 8 we show again the contours of the growth rate in the $k-m$ plane as in Fig. 5 but this time comparing $k_{\max }(m)$, i.e., the wave number corresponding to the maximum growth rate (depicted by solid bold lines) with (i) $k_{0.65 \pi}(m)$, i.e., the wave number for which the phase difference is equal to the optimal phase difference for $m=0$ and $L_{d}=\infty, 0.65 \pi$ (dashed lines), and (ii) $k_{\text {equal }}(m)$ (dotted lines). For all the values of the Rossby deformation radius, the unstable regions enclose the dotted lines, i.e., where the two isolated CRWs have the same phase speed. This confirms that the two isolated CRWs must have a similar phase speed to have phase locking since the change in phase speeds due to the presence of the other CRW is small. For $L_{d}=\infty$ [Fig. 8(a)] we observe that the position of the maximum of the growth rate almost fully corresponds to where the phase difference is equal to $\Delta \epsilon=0.65 \pi$. This shows that the introduction of the background shear does not qualitatively modify the phase locking. However, the discrepancy between the dashed and the solid lines increases as much as $L_{d}$ is reduced. Therefore, the deformation radius significantly modify the value of the optimal phase difference.

To make this point more evident, we illustrate in Fig. 9 the value of the phase difference corresponding to the maximum growth rate $\Delta \epsilon_{\mathrm{opt}}$. The black line discriminates between helping $\left(-\frac{\pi}{2}<\Delta \epsilon<\frac{\pi}{2}\right)$ and hindering $\left(\frac{\pi}{2}<\Delta \epsilon<\frac{3}{2} \pi\right)$ configurations. When the Rossby deformation radius is infinite, the influence of the background shear is weak. However, the optimal phase difference is slightly diminished by adding both a favorable or an adverse shear. This means that either the influence of one CRW on the other's phase speed is stronger or the two isolated CRWs have a closer value of the phase speed, so they need less hindering effect to phase lock. The former case occurs for $m>0$ since also the influence on the each other's phase speed is proportional to $\gamma$, which is larger at small wave numbers [see Fig. 6(b)]. For $m<0$ instead the latter case occurs since the difference between the two phase speeds of the isolated CRWs is smaller.

However, the dependence of $\Delta \epsilon_{\mathrm{opt}}$ on $L_{d}$ is more significant, in particular for positive $m$. We observe a general decrease in the optimal phase difference. This is due to the fact that the interaction is weaker when $L_{d}$ is finite, as seen in Fig. 7(b). For a favorable background shear the two CRWs need a helping configuration to phase lock if $L_{d}$ is very small. This occurs since the two CRWs lose their counterpropagative character for small $L_{d}$, as observed in Fig. 4(b). Thus, to have phase locking, each CRW needs help from the other wave to withstand the mean flow.

\section{NONLINEAR SIMULATIONS}

In this section we conduct nonlinear simulations of the quasi-Rayleigh profile which have been presented in Sec. II A. The simulations will be set up to investigate the breakdown of a shear layer, treated as a filament with unit PV in its interior and zero PV on its exterior, subjected to an externally forced global background shear. The linear phase evolution of this setup should follow that of the system analyzed in Sec. II provided that the PV jump across the filament edges and the streamwise velocities along each filament edge are equivalent in both model systems.

\section{A. Governing equations and numerical setup}

\section{Governing equations}

The nonlinear equations of motion we solve are

$$
\left[\frac{\partial}{\partial t}+(\tilde{m} y+u) \frac{\partial}{\partial x}+v \frac{\partial}{\partial y}\right] q=-v_{8} \nabla^{8} q,
$$



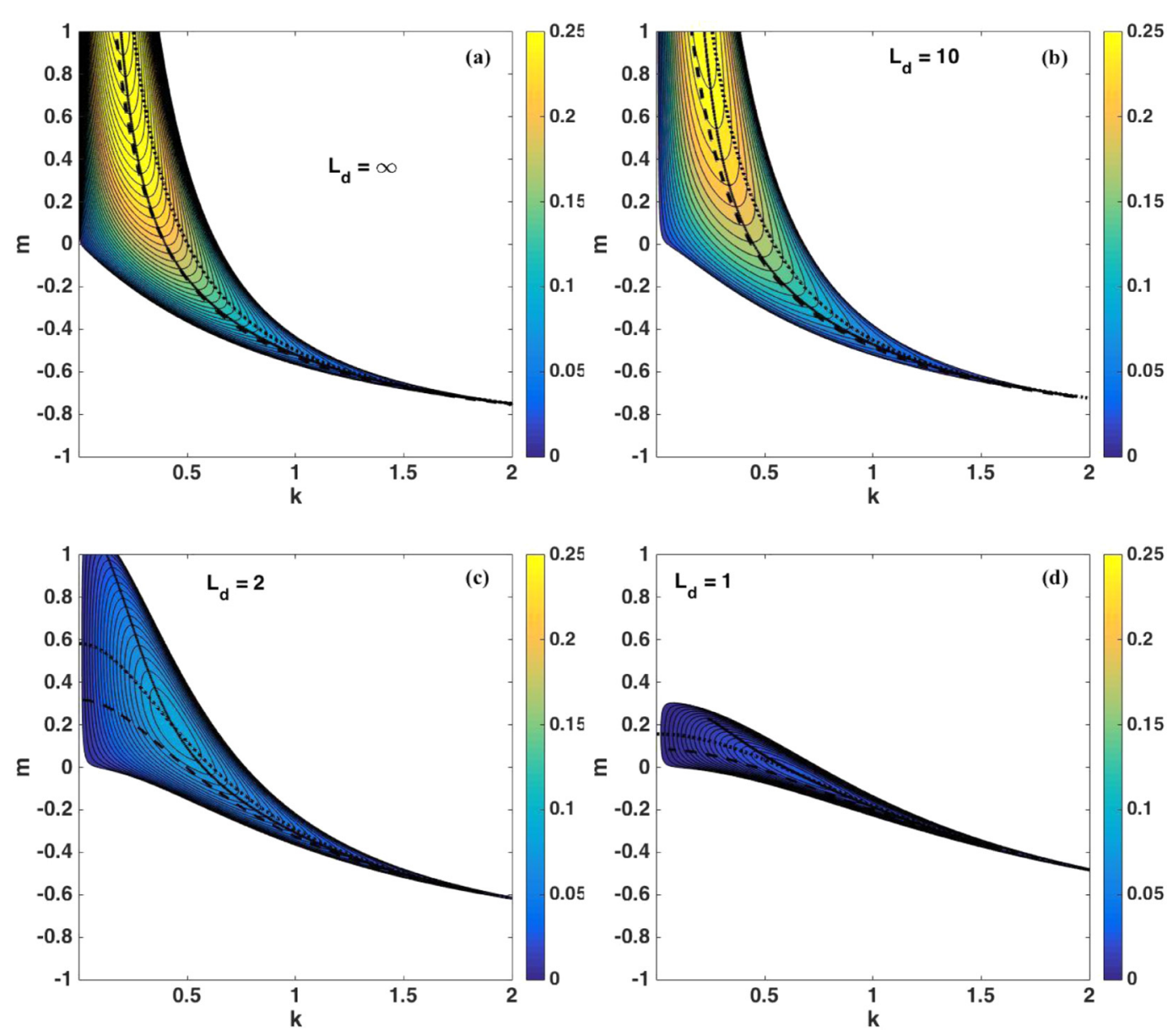

FIG. 8. Growth rate $\omega_{i}$ in the plane $k-m$ for (a) $L_{d}=\infty$, (b) $L_{d}=10$, (c) $L_{d}=2$, and (d) $L_{d}=1$. The solid line represents $k_{\max }(m)$, the dashed line represents $k_{0.65 \pi}(m)$, and the dotted line represents $k_{\text {equal }}(m)$.

where

$$
q \equiv \nabla^{2} \psi-L_{d}^{-2} \psi, \quad u \equiv \frac{\partial \psi}{\partial y}, \quad v \equiv-\frac{\partial \psi}{\partial x} .
$$

We have set the planetary rotation parameter $\beta$ to zero. The background shear ( $\tilde{m} y)$ is given as immutable and is the only source of continual external forcing. This is in addition to the original filament profile with which we initiate the simulation. Deviations of this flow are given by the initial form adopted for $q$. We will consider rounded models of the basic forms described in Eq. (3) (discussed further below). Because regular viscosity is set to zero, in order to dissipate enstrophy or energy at the smallest scales of the simulation we have found an eighth-order superviscosity operator $-v_{8} \nabla^{8}$ to the PV evolution equation as the most suitable option (also see Appendix A). However, it should be noted that other lower orders have been tested with no significant changes in the results, but the energy cascades were less visible in the spectra. The connection between $\tilde{m}$ and the value of $m$ used in our analysis in Sec. II will be clarified further in Sec. IV A 2.

\section{Simulation setup}

We must set up the initial filament profile as a streamwise uniform solution of the fundamental equations. The velocity field of the classical setup found in Sec. II has exponential divergences as 


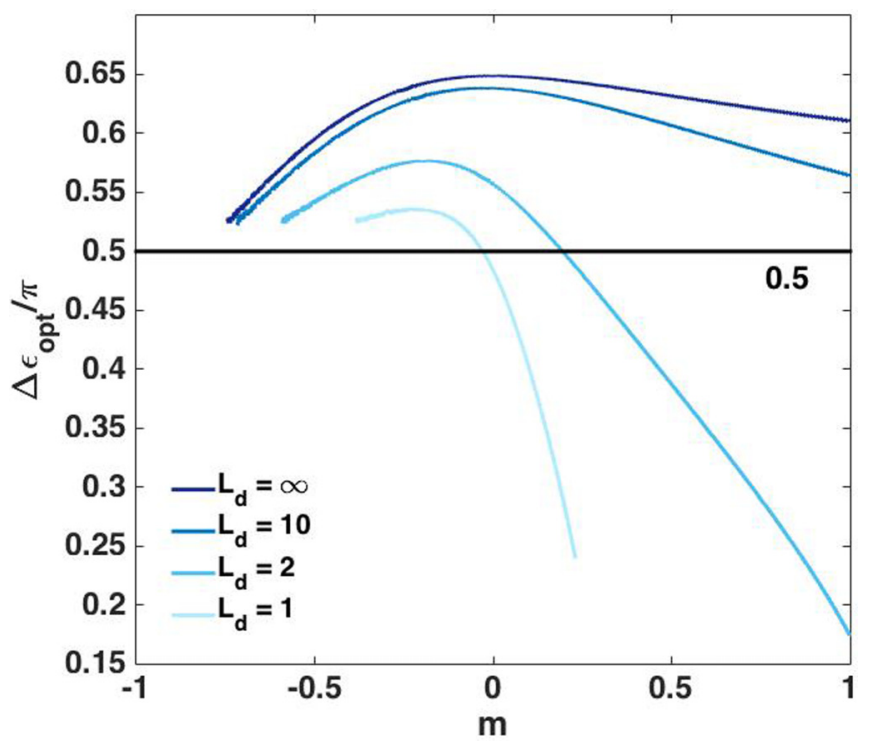

FIG. 9. Phase difference corresponding to the maximum growth rate $\frac{\Delta \epsilon_{\max }}{\pi}$ vs wave number for $m=0$ and different values of $L_{d}$. The black line represents the limit between the helping and the hindering configuration.

$|y| \rightarrow \infty$. We must then be careful in choosing the initial velocity profile since if the horizontal velocities get very large, we invariably run into numerical stability issues, especially for runs in which the breakdown is violent, exhibiting a high degree of spreading in the shearwise direction. As per our considerations at the beginning of this section, in order to connect to the filaments examined in the linear analysis, we have to make sure that (i) the jump in PV across the filament boundaries is the same (in this case this jump in PV is $\Delta q=1$ ) and (ii) the streamwise velocities on the two boundaries must be the same as well. Constructing this consistently will determine a connection formula between $\tilde{m}$ and $m$. We approach in the following way. We divide the PV as one being composed of a piecewise constant quasi-Rayleigh profile plus the aforementioned globally forced background uniform shear $\tilde{m} y$. This quasi-Rayleigh PV is denoted by $Q_{q R}$ and is the solution of

$$
\left(\nabla^{2}-\frac{1}{L_{d}^{2}}\right) \Psi_{q R} \equiv Q_{q R}= \begin{cases}0 & \text { for }|y|>1 \\ -1 & \text { for }|y| \leqslant 1\end{cases}
$$

where $\Psi_{q R}$ is the corresponding stream function of this steady state. A comparison of the right-hand side of Eq. (16) to the PV adopted in Sec. II, i.e., as summarized in Eq. (3), shows that the two show the same jump in PV across their filament boundaries at $y= \pm 1$. The mean streamwise velocity of this quasi-Rayleigh profile is given as

$$
U_{q R}(y)= \begin{cases}u_{0} e^{(1-y) / L_{d}} & \text { for } y>1 \\ u_{0} \sinh \left(\frac{y}{L_{d}}\right) / \sinh \left(\frac{1}{L_{d}}\right) & \text { for }|y| \leqslant 1 \\ -u_{0} e^{(y+1) / L_{d}} & \text { for } y<-1,\end{cases}
$$

with $u_{0}=\frac{L_{d}}{1+\operatorname{coth}\left(\frac{1}{L_{d}}\right)}$. To complete the correspondence between the flow setup here and that considered in Sec. II, we must make sure that the velocities at $y= \pm 1$ are equal to one another. This means setting $\pm \tilde{m}+U_{q R}( \pm 1)=U( \pm 1)$, where $U$ is the streamwise velocity found in Eq. (2). Owing to the symmetry of the profile with respect to $y=0$, this amounts to

$$
\tilde{m}+\frac{L_{d}}{1+\operatorname{coth}\left(\frac{1}{L_{d}}\right)}=L_{d}(m+1) e^{-1 / L_{d}} \sinh \left(\frac{1}{L_{d}}\right) .
$$


Simplifying this relationship for $\tilde{m}=\tilde{m}\left(m, L_{d}\right)$, we find

$$
\tilde{m}\left(m, L_{d}\right)=\frac{m L_{d}}{1+\operatorname{coth}\left(\frac{1}{L_{d}}\right)},
$$

which completes the correspondence we have sought to establish.

All simulations are then initially seeded with these piecewise constant quasi-Rayleigh filament solutions, i.e., $q(t=0)=Q_{q R}$. We do this by utilizing a rounded approximation of $Q_{q R}$ given by $\tilde{Q}_{q R}$, where

$$
\tilde{Q}_{q R}(\epsilon)=-\frac{1}{2}\left[\tanh \left(\frac{y+1}{\varepsilon}\right)-\tanh \left(\frac{y-1}{\varepsilon}\right)\right],
$$

in which $\varepsilon$ controls the tightness of the transition across the filament boundaries. A cursory inspection shows that

$$
\lim _{\varepsilon \rightarrow 0} \tilde{Q}_{q R}(\epsilon) \rightarrow Q_{q R}
$$

In order to resolve the PV transition at $y= \pm 1$ we use at least five to seven grid points, which generally means having values of $\varepsilon=0.05$ (keeping in mind a typical grid spacing of about $\Delta y \approx$ $0.05)$. In addition to this initial filament we introduce an additional amount of white noise in the PV field. This noise is constrained to be nonzero in a region $|y|<2$. At maximum, the noisy PV field has an amplitude which is about $25 \%$ of the value inside the filament.

Appendix A contains a description of the pseudospectral numerical method we employ to solve this system. We find that the linear phase evolution of this setup is indeed practically identical to the one predicted for the model described in Sec. II, which largely contributes to validating the use of this numerical method. Further details may be found in Appendix B.

\section{B. Results}

In this section the results of the nonlinear simulations are reported. We analyze (i) the potential vorticity fields (Sec. IV B 1), (ii) the momentum thickness growth (Sec. IV B 2), and (iii) the perturbation kinetic energy (Sec. IV B 3). In our simulations the domain size is settled to $L_{x}=49.3$ and $L_{y}=60.0$ ( $L_{y}=30.0$ for $L_{d} \leqslant 2$ for ensuring numerical stability). A random disturbance is added to the initial field as explained in Sec. IV A 2.

\section{Potential vorticity fields}

In Fig. 10 the potential vorticity fields showing the nonlinear evolution of the classical Rayleigh profile (i.e., $m=0$ and $L_{d}=\infty$ ) are illustrated at different times from $t=8$ [Fig. 10(a)] to $t=$ 150 [Fig. 10(f)]. Four vortices are created by the initial disturbance [Figs. 10(b) and 10(c)]. These vortices pair with each other, causing the growth of the shear layer [Figs. 10(d) and 10(e)]. Finally, they combine to form one single rotating vortex with filamentary arms [Fig. 10(f)]. The vortex pairing is the main cause of the spreading of the mixing layer [36]. This behavior is typical of two-dimensional mixing layers and the reader is referred to the review of Ho and Huerre [37].

In Fig. 11 we add a uniform adverse shear to the Rayleigh profile. In particular, the slope of the background shear is set equal to $m=-0.2$, while the Rossby radius of deformation is still $L_{d}=\infty$. The initial disturbance generates again four vortices [Fig. 11(b)]. The adverse shear background increases significantly both the number and the resistance of the filamentary structures around the vortices [Fig. 11(c)]. The vortex pairing is weakened by these filaments. The reduction of the vortexmerging process indeed hinders the growth of mixing layer. For this reason, the vortices size does not grow as much as without adverse shear (i.e., $m=0$ ) [see Fig. 11(d)].

In Fig. 12 no uniform shear is added to the Rayleigh profile (i.e., $m=0$ ), but a finite value of the Rossby radius of deformation is introduced $\left(L_{d}=2\right)$. There are four vortices created by the initial random disturbance [Figs. 12(b) and 12(c)]. In contrast to $m=0$, the vortices do not merge with 

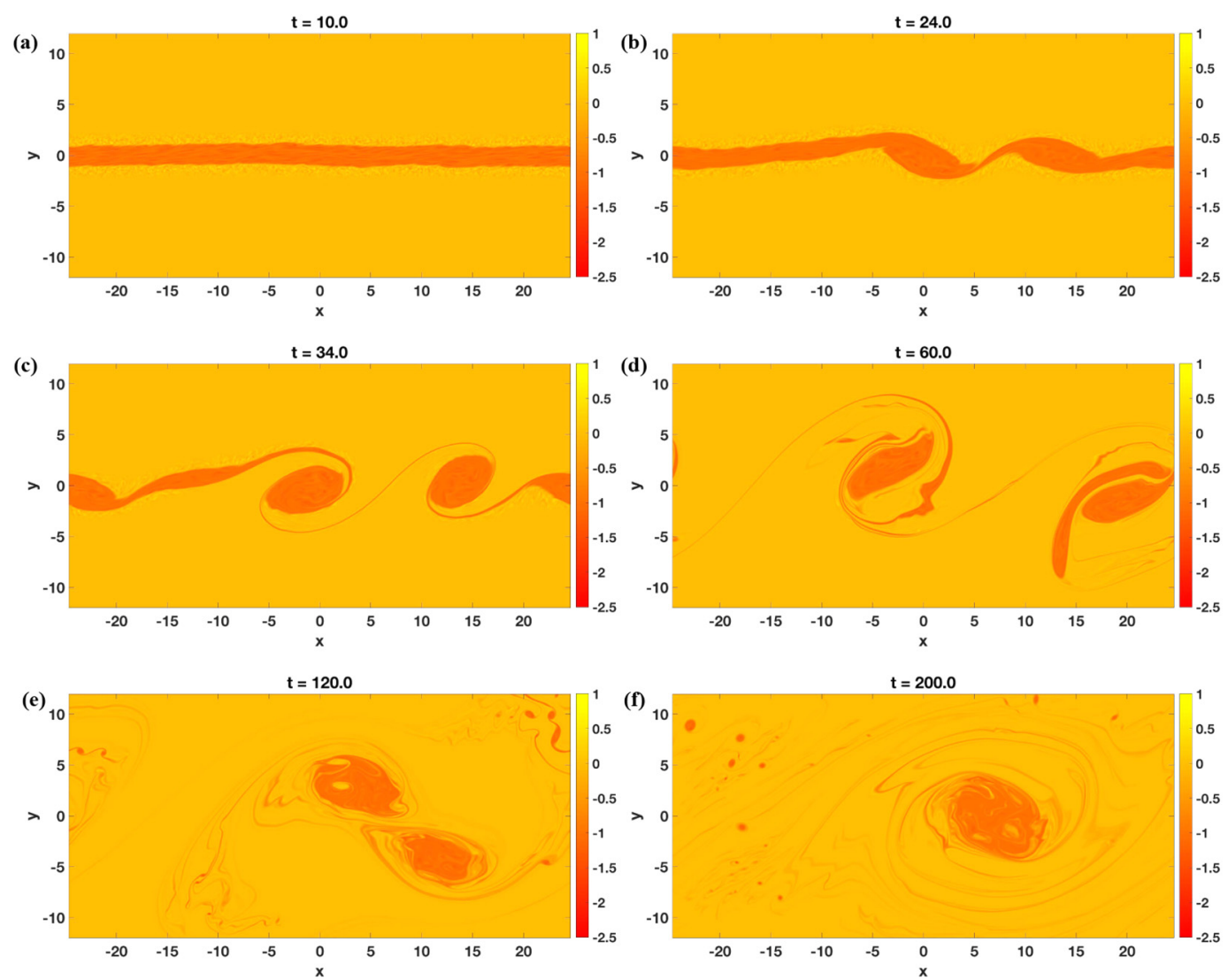

FIG. 10. Potential vorticity fields for $m=0$ and $L_{d}=\infty$ at different times (a) $t=10$, (b) $t=24$, (c) $t=34$, (d) $t=60$, (e) $t=120$, and (f) $t=200$.

each other but are slowly rotating [Fig. 12(d)]. Since the vortex merging is absent, the mixing layer does not grow as much as for $L_{d}=\infty$.

\section{Momentum thickness}

In this section the spread of the mixing layer is analyzed. Therefore, a proper definition for the momentum thickness $\theta$ is needed. We define it as

$$
\theta(t)=\int_{-L_{y}^{*} / 2}^{L_{y}^{*} / 2} \frac{\bar{u}(y, t)-U_{1}(y)}{U_{2}(y)-U_{1}(y)}\left(1-\frac{\bar{u}(y, t)-U(1)}{U_{2}(y)-U_{1}(y)}\right) d y,
$$

where $L_{y}^{*}=0.8 L_{y}$ is a vertical length around the shear layer to avoid contamination from the boundaries, $\bar{u}$ is the horizontal velocity averaged along the $x$ direction, and $U_{1}(y)$ and $U_{2}(y)$ are

$$
\begin{aligned}
& U_{1}(y)= \begin{cases}U(y) & \text { for } y>1 \\
U(1) & \text { for } y \leqslant 1,\end{cases} \\
& U_{2}(y)= \begin{cases}U(-1) & \text { for } y>-1 \\
U(y) & \text { for } y \leqslant-1,\end{cases}
\end{aligned}
$$

respectively. If $m=0$ and $L_{d}=\infty$ (i.e., the classical Rayleigh profile), $U_{1}$ and $U_{2}$ are constant and then Eq. (21) becomes the widely known equation for the momentum thickness $[36,38]$. 

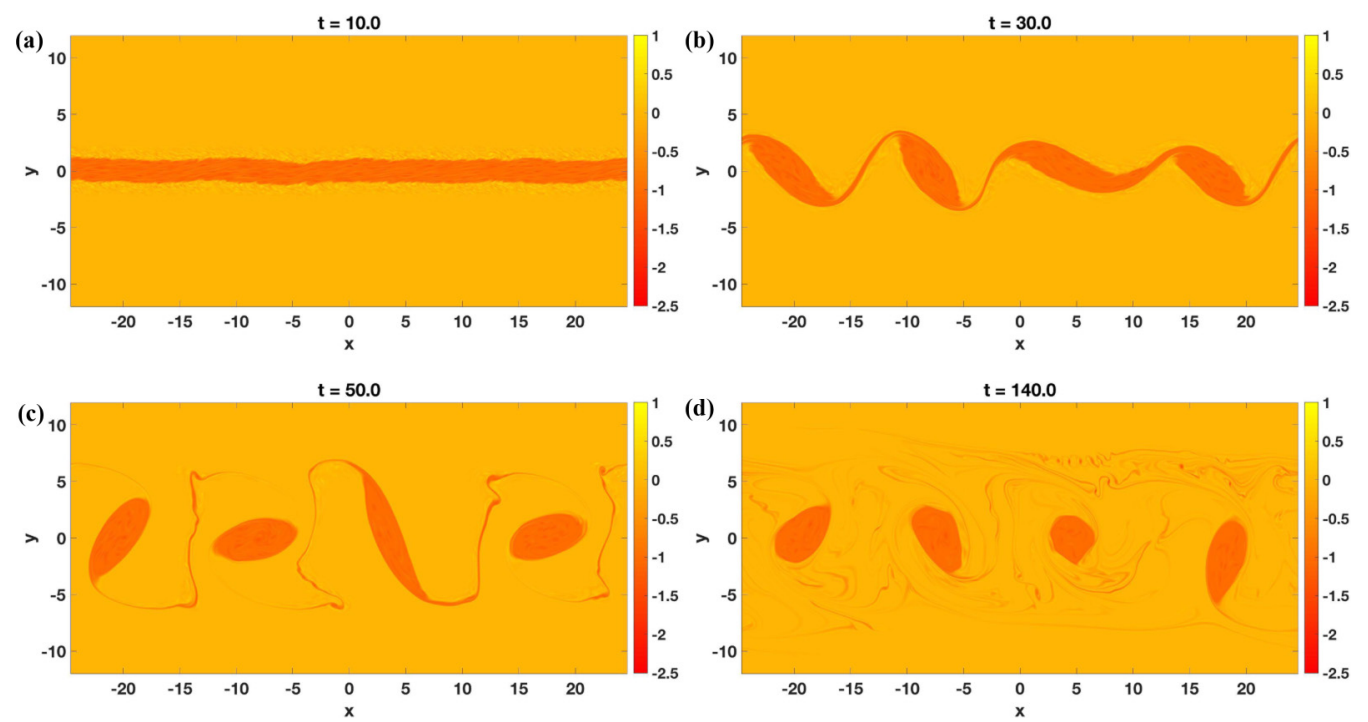

FIG. 11. Potential vorticity fields for $m=-0.2$ and $L_{d}=\infty$ at different times (a) $t=10$, (b) $t=30$, (c) $t=50$, and (d) $t=140$. Note that at late times $(t=140)$ small-scale filaments appear to undergo a secondary transition into coherent vortices.

In Fig. 13(a) the temporal evolution of the momentum thickness is shown for $L_{d}=\infty$ and different uniform shear $m$. Interestingly, the maximum spread occurs without the background shear (i.e., $m=0$ ). For negative $m$ we have seen in Fig. 11 that the vortex merging is significantly weakened. This is expected to result in a corresponding weakening of the mixing-layer growth. However, this dampening is present for positive $m$ also in which the vortex pairing indeed occurs. For this case the momentum thickness cannot significantly diffuse outside the initial shear layer
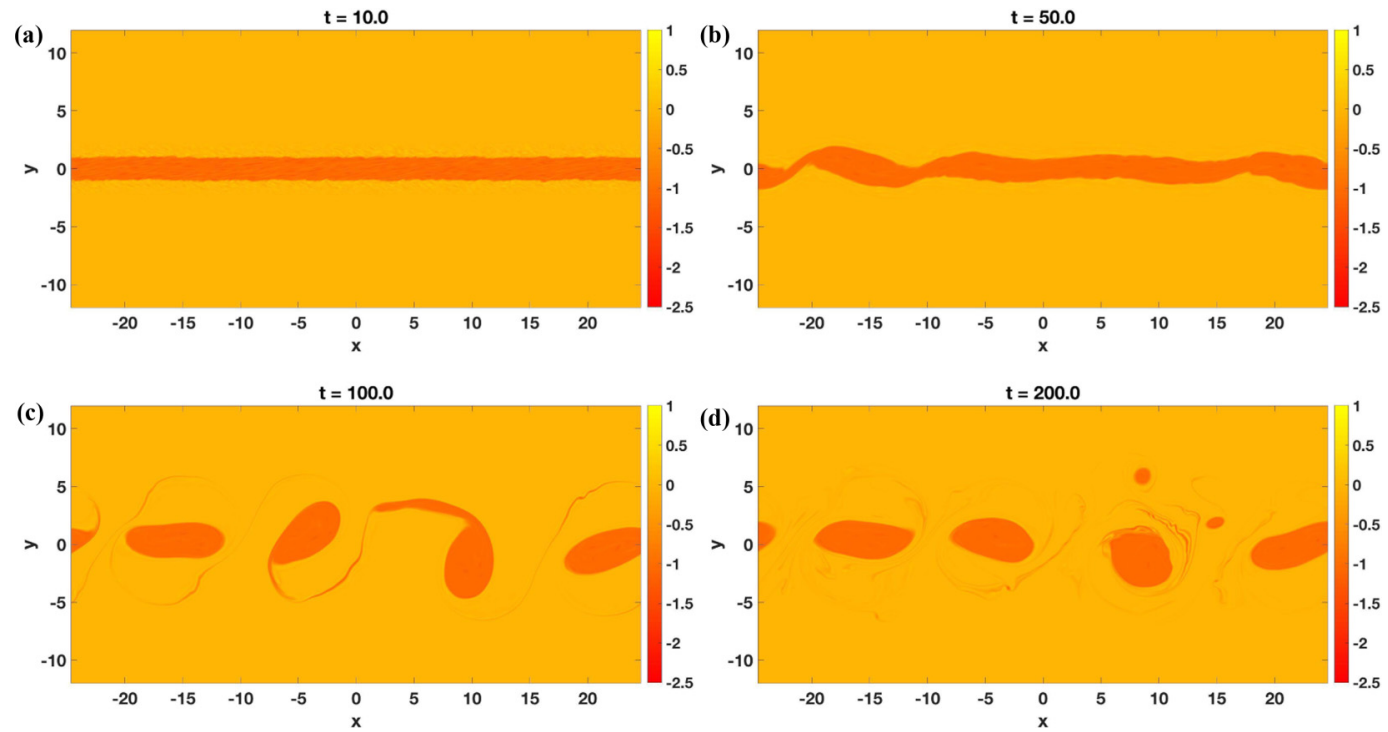

FIG. 12. Potential vorticity fields for $m=0$ and $L_{d}=2$ at different times (a) $t=10$, (b) $t=50$, (c) $t=$ 100 , and (d) $t=200$. Similar to Fig. 11 , torn shreds of vorticity appear to roll into smaller satellite coherent vortices at late times. 

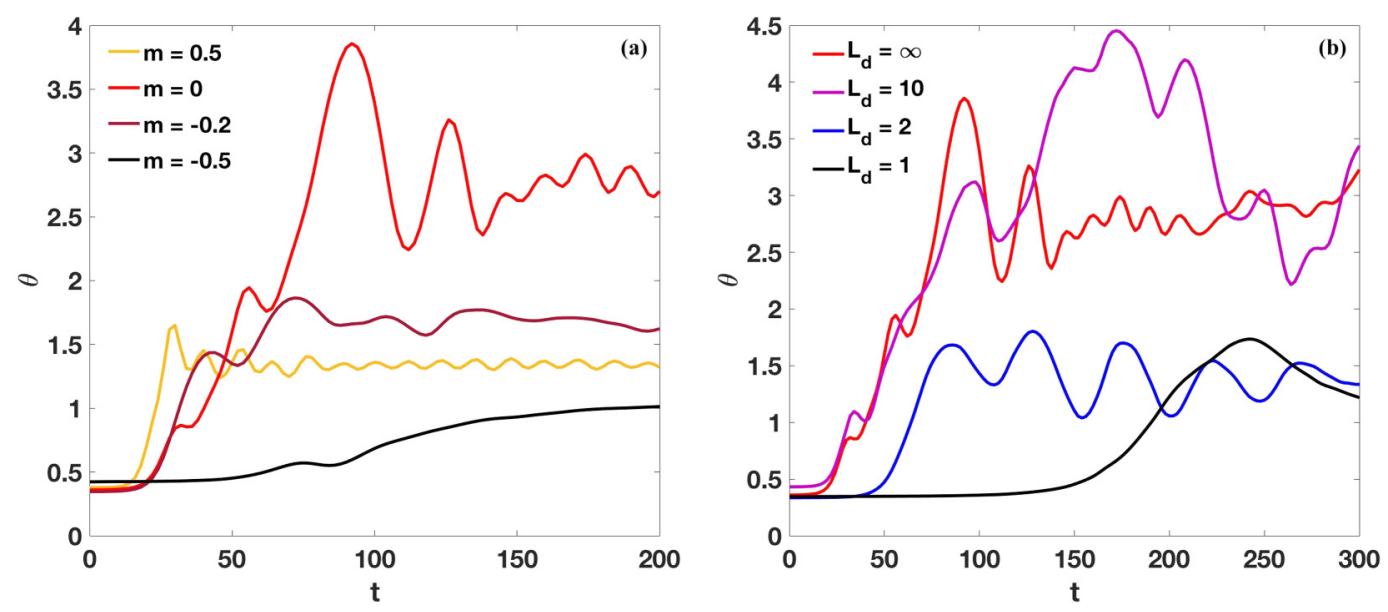

FIG. 13. Temporal evolution of the momentum thickness $\theta$ for (a) $L_{d}=\infty$ and several values of $m$ and (b) $m=0$ and several values of $L_{d}$.

due to the uniform background shear which acts to confine filaments in the shearwise direction by rapidly stretching them out and orienting them into the shearwise direction [39]. Particularly the spreading is hindered by the increased momentum of the sandwiching layers.

Furthermore, the temporal growth of the mixing layer is illustrated in Fig. 13(b), which introduces a finite value of $L_{d}$ in the absence of the background shear. As the Rossby radius of deformation decreases, the mixing-layer spread is damped. This is not surprising since we observed in Fig. 12 that a finite value of $L_{d}$ eliminates the vortex pairing which is the main cause of the growth of mixing layers [36].

\section{Perturbation kinetic energy: Temporal evolution and spectra}

The temporal evolution of the perturbation kinetic energy averaged along both the $x$ and $y$ directions, $K$, is shown in Fig. 14 for $L_{d}=\infty$ and different values of $m$ [Fig. 14(a)] and for $m=0$ and different values of $L_{d}$ [Fig. 14(b)]. At the beginning the effect of background shear
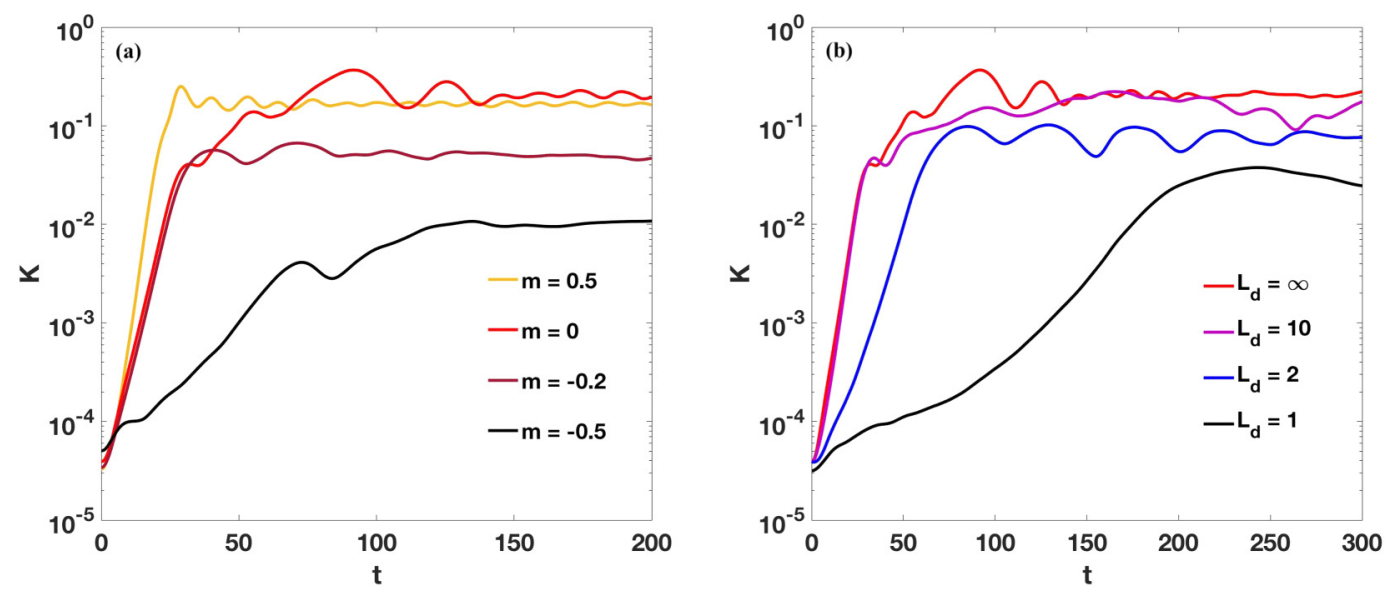

FIG. 14. Spatially averaged perturbation kinetic energy for (a) $L_{d}=\infty$ and several values of $m$ and (b) $m=0$ and several values of $L_{d}$. The strength of the turbulence is growing with increasing $m$ and diminishing $L_{d}$. Note that the domain sizes are $L_{x}=49.2928$ and $L_{y}=60$ or $L_{y}=30$ (when $L_{d} \leqslant 2$ ). 

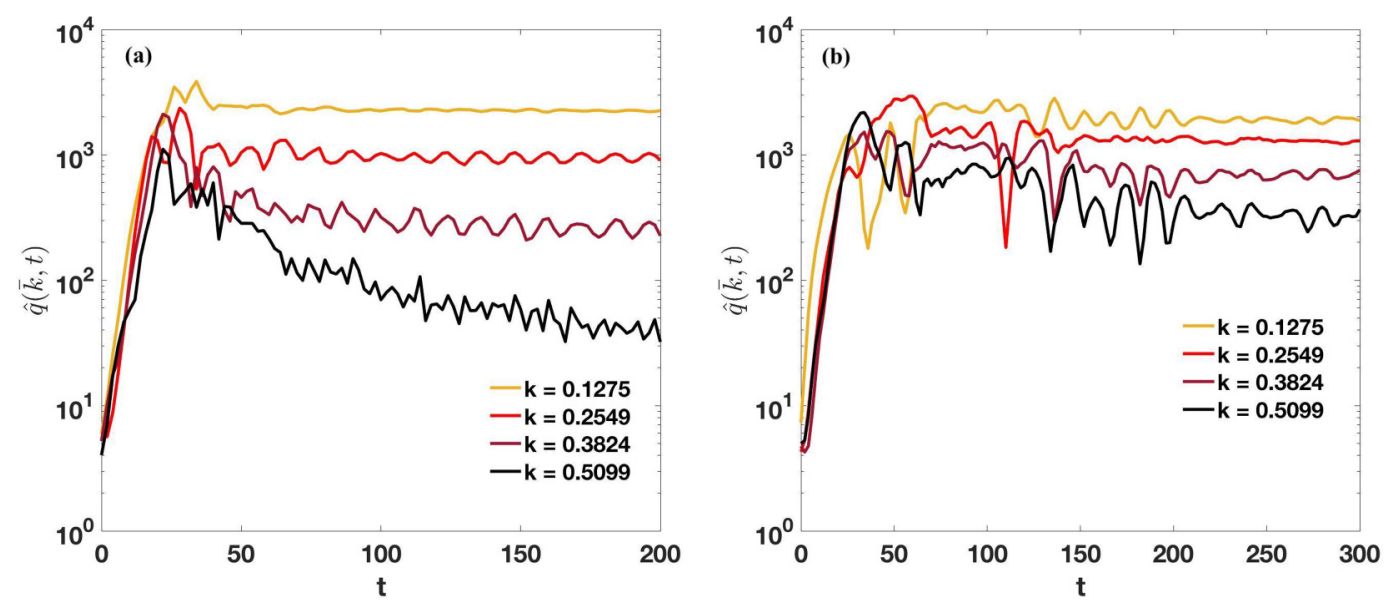

FIG. 15. Fourier transform of the potential vorticity $\hat{q}(\bar{k})$ with $k$ equal to $k=0.1275$ (yellow), $k=0.2549$ (red), $k=0.3824$ (dark red), and $k=0.5099$ (black) for $L_{d}=\infty$ and (a) $m=0.5$ and (b) $m=0$.

is in agreement with the linear theory, as can be seen in Fig. 14(a): If the value of $m$ is decreased, the strength of the perturbation increases. However, once nonlinear effects start to be significant, a discrepancy with the linear theory is noticed. While the dampening effect due to an adverse shear is still present, a cooperative shear hinders the growth of $K$ as well once saturation is reached. The additional boost in energy to the case without background shear is given by secondary growing modes triggered by the initial unstable mode. This is consistent with the fact that the cutoff number for $m=0$ is larger than for $m=0.5$ [see Fig. 3(a)] with a wider range of unstable wave numbers.

The nonlinear effect of $L_{d}$ on the temporal evolution of $K$ is in agreement with the linear theory [Fig. 14(b)]. As the Rossby radius of deformation gets smaller the perturbation kinetic energy decreases. The kinetic energy is partially "wasted" along the transversal direction, thus there is less available energy to feed the growth of the mixing layer along the vertical direction.

In Fig. 15 the $y$-integrated Fourier transform along $x$ of the potential vorticity $\hat{q}(k, t)$ is illustrated for $L_{d}=\infty$ and $m=0.5$ [Fig. 15(a)] and $m=0$ [Fig. 15(b)]. In this manner we can observe in detail which wavelengths are triggered. In our simulations we initially trigger a mode with wavelength $\frac{2 \pi}{k}$, where $k=0.1275$. For $m=0.5$ this mode dominates both the linear response and the turbulent state. For $m=0$ the initial instability triggers modes with different wave numbers, such as $k=0.2549$ and $k=0.5099$. Then the energy of the final saturated state is not just due to the energy of the initial unstable mode, but several modes play a role to reach this value. This overall picture involving only a few mode interactions is consistent with previous studies examining the growth and saturation of mixing layers in porous media using both weakly nonlinear and numerical methods [40]. This confirms our previous hypothesis that the appearance of secondary modes, favored for $m=0$ by the broader range of unstable wave numbers [see Fig. 3(a)], enhances the level of turbulent kinetic energy for $m=0$ with respect to $m=0.5$ [see Fig. 14(a)].

The time-averaged spectra of the perturbation kinetic energy $K$ are reported in Fig. 16 for $L_{d}=\infty$ and several values of $m$ [Fig. 16(a)] and $m=0$ and several values of $L_{d}$ [Fig. 16(b)]. Dashed lines depict the slope of the spectra in the inertial range where the enstrophy cascade occurs. In both figures the slope is steeper than the theoretical value of -3 predicted by Kraichnan [1] for steady forced-dissipative 2D turbulence. In particular, for $L_{d}=\infty$ the slope is between -3.65 and -3.67 for all the values of $m$. These values are very close to the $-\frac{11}{3}$ previously reported in other two-dimensional turbulent mixing-layer calculations $[28,38]$ and as such we view the spectral correspondence, across the whole slew of $L_{d}=\infty$ models examined here, as further validation of our numerical solution methods. From a kinematic point of view, Gilbert [27] has explained that such value is due to the spiral filamentary structures that grow around coherent vortices [see 

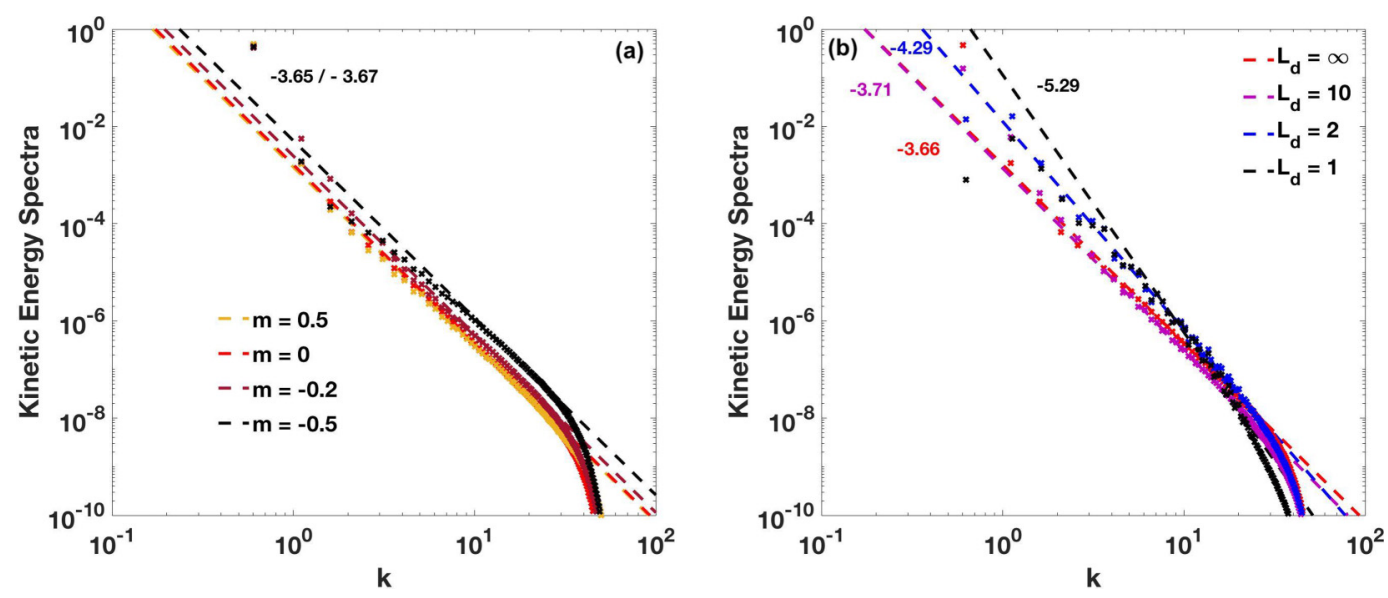

FIG. 16. Time-averaged kinetic energy spectra for (a) $L_{d}=\infty$ and several values of $m$ and (b) $m=0$ and several values of $L_{d}$.

Fig. 10(f), for instance] which are themselves characterized by vorticity gradients perpetuating to ever-smaller scales in the spaces between the surviving large-scale coherent structures (see further discussion below). Also, as indicated in Fig. 16(a), we find it significant that the $-\frac{11}{3}$ spectrum persists irrespective of the globally imposed constant shear.

Finally, for a finite value of $L_{d}$ the slope of the spectra significantly increases. For $L_{d}=1$ the slope value increases up to -5.29 . Previous authors [31,41-43] found such steep spectra for the enstrophy cascade range (normally between -4 to -6 ) due to intermittency of the enstrophy transfer towards small scales. The enstrophy cascade is fueled by vorticity gradients $[4,8,44]$. The significant reduction of filaments around the vortices when $L_{d}$ is finite [see Fig. 11(d)] shows how the vorticity gradients are weakened around the vortices. Therefore, the direct enstrophy cascade is strongly hindered by a small $L_{d}$ and this is revealed by the steepest spectra shown in Fig. 16(b). We discuss this further below in the next section.

\section{Interpretation}

The features examined in the previous sections lead us to adopt the following interpretation inspired in part by the picture of self-similar coherent vortex production [31,32], while bearing in mind that the behavior of a turbulent mixing layer cannot be fully explained by filaments breakdown only. In stationary driven-decaying $2 \mathrm{D}$ turbulence calculations at $L_{d}=\infty$, e.g., as recently reviewed by Boffetta and Ecke [4], vortices are produced at some rate at some given length scale. A direct enstrophy cascade ensues as these coherent vortices undergo structural breakdown that generate filaments that become elongated, bent, and sheared and eventually fully populate the spaces between the (quasi)coherent structures of the injection scales. Ultimately, this process cascades down to the dissipation scales giving rise to the nearly $k^{-3}$ energy spectra predicted by Kraichnan [1] to characterize these flows.

Of course, current best resolved numerical simulations [45] show that the spectrum takes on the form $k^{-3-\delta}$ with $\delta \approx 0.65$. Boffetta and Musacchio's study [45] involved conducting a series of numerical experiments with increasing resolution and showed that $\delta$ progressively gets smaller as the resolution and Reynolds number increase. They conclude that this system ought to asymptotically yield $\delta \rightarrow 0$ as $\operatorname{Re} \rightarrow \infty$.

The ability to shred apart vortices is directly a function of the relative distance between, say, two vortices and their mutual ability to influence or advect their edges which is in turn a function of $L_{d}$ : Larger values of $L_{d}$ implies that vortex structures can induce flow at longer range. Obviously, 
for a given separation of two given coherent vortices of given size, filament production should be preferentially promoted in models where $L_{d}$ is largest. With this physical view, it therefore seems reasonable to suppose that spatially dense filaments with strong spatial gradients become rarer in models where $L_{d}$ decreases.

We view the features of the mixing layer within this same framework, but we read its implications in the following way: The mixing layer has no steady production rate of vortices but has instead a short duration production of vortices owing to the roll-up or breakdown of the original layer itself. This generates filaments which wind up in response to the velocity field of the aggregate vorticity distribution and, when applicable, the background imposed uniform vorticity. In cases when several coherent vortices are born from the primary breakdown, copious filamentary structure is produced as in all of the $L_{d}=\infty$ cases we examined (see, e.g., Figs. 10 and 11). Within these subset systems, coherent vortices tend to merge when the background shear is zero which in turn further produces more filamentary structures both inside and outside the resulting merged structure; this is especially evident in the latter stages of development as shown in Fig. 10(d). In both cases, filaments are seen to also roll up and generate smaller-scale coherent structures as well, suggesting that a selfsimilar process is at play. In this sense, it appears that the filaments are not nearly as space filling as they are in the classical stationary forced-dissipated $2 \mathrm{D}$ turbulence systems, with its usual $k^{-3}$ direct enstrophy cascade spectra, since coherent vortices appear to be produced alongside spiraling filaments at ever decreasing spatial scales [29]. While these coherent structures remain stable to destruction, vorticity stays locked within them, choking off the production of more filaments at smaller scales [46]. It is important to note that a flow that only yields a self-similar distribution of vortices at all scales down to the dissipation scales will exhibit an energy spectrum of the form $k^{-5}$ (see Refs. [30,31]). We can think of this idealized self-similar vortex system as one end-member state, with the other end-member being steady forced-dissipated 2D turbulence with its space-filling spiraling vortex filaments. In this sense, and very much in line with Gilbert's thinking [27], we rationalize the $k^{-11 / 3}$ spectral slope of these mixing-layer experiments to be a reflection of the fact that they, as a setting, lie in between these two end-member states since both self-similar coherent structures are present alongside ever tightly spiraling vortex filaments.

The simulations with $L_{d}$ small like those shown in Fig. 12 may be interpreted in a similar vein. These mixing layers also undergo breakdown, but their coherent child PV structures are relatively stable. This stability is due entirely to the fact that individual coherent structures have more limited dynamical reach compared to their larger $L_{d}$ counterparts. That is to say, the flow induced by a coherent PV structure is weaker and greatly restricted in range compared to a similar structure with a larger value of $L_{d}$. In these smaller $L_{d}$ settings, unless individual coherent PV structures get very close one another, vortex-vortex mergers cannot occur as readily as they do in $L_{d} \rightarrow \infty$ conditions. With merging events becoming infrequent, fewer filaments get produced and most of the PV remains locked within these larger-scale structures. This gives rise to the steepness of the energy spectra observed in these cases, which increasingly resembles the idealized self-similar vortex end-member case with its characteristic $k^{-5}$ energy spectrum.

\section{CONCLUSION}

In this work we have revisited by means of the KW perspective the linear and nonlinear stability of quasigeostrophic vortex filaments under the influence of a background shear. These filaments were modeled by a family of quasi-Rayleigh profiles subject to a uniform shear $m y$.

Through the KW perspective, the instability of the quasi-Rayleigh profiles is viewed as the interaction of two counterpropagating Rossby waves created at the two PV edges (i.e., where the $\mathrm{PV}$ is discontinuous).

We confirmed that an adverse (favorable) shear stabilizes (destabilizes) the flow, as seen by Dritschel [5]. Owing to the KW framework, we observed that the optimal phase-locking configuration is shifted towards smaller (larger) wave numbers if $m$ is positive (negative), while the interaction between the two CRWs is totally independent of the background shear. Phase locking 
occurs in a range of wave numbers in which the interaction is more (less) favored for positive (negative) $m$ than when the background shear is absent. The introduction of a finite value of $L_{d}$ has a stabilizing influence on the Rayleigh profile independent of the presence of a background shear. As the Rossby deformation radius $L_{d}$ decreases, the interaction between the two CRWs is weakened. This agrees with the common understanding that QG effects diminish the reach of the edge waves.

We have examined the nonlinear breakdown of a vortex filament in the nominally infiniteReynolds-number limit by using a superviscosity operator to drain power that builds up on individual grid scales due to turbulent cascade through the inertial regime. In the simulations, the quasi-Rayleigh profile is again used to model the vortex filaments, while the background shear is viewed as immutable and a continuous source of external forcing. We have validated our numerical code and model setup by comparing our linear predictions to our numerical simulations during the linear growth phase. We have demonstrated the usefulness of the KW perspective through this equivalence (Sec. IV). The KW viewpoint says that at the two edges of the mixing layer, only the PV jump and the mean flow evaluated at these edges determine the stability of the mixing layer. Indeed, the mean streamwise velocity fields in the background shear enhanced quasi-Rayleigh profile we have adopted and the profile analyzed in the linear analysis are very different. However, the two flow fields share the same two core characteristics, i.e., the PV jumps across strip edges and the total mean flow at these edges are equivalent in both profiles. Both profiles are shown to yield the same growth rates.

With regard to the quality of the mixing layer's development and breakdown, we found several properties: A negative background shear hinders the vortex pairing and then the growth of the mixing layer while with a positive background shear the growth mechanism is still obstructed, but this is due to the increased momentum of the external layers. A finite value of the Rossby radius of deformation also hinders the vortex pairing; then the mixing-layer growth is maximized with a configuration with an infinite $L_{d}$. While the deformation radius has a similar influence on linear and nonlinear analyses, we observed discrepancies between these analyses concerning the impact of the background shear on the turbulence strength. In particular, they differ for a positive $m$ since in the nonlinear simulations the most energetic configuration is without a background shear, while for the linear analysis the gravest growth rate was for $m=0.5$. For zero background shear additional modes are subharmonically activated by the disturbance, while this does not occur for $m=0.5$ since the range of unstable wavelengths is narrower.

When $L_{d}$ is infinite, the slope of the enstrophy cascade in the kinetic energy spectra is very close to the value of $-\frac{11}{3}$, typical of $2 \mathrm{D}$ turbulent mixing layers [28,38]. This value for the slope is intermediate between -3 (predicted by Kraichnan [1] for forced-dissipated 2D turbulence systems) and -5 (found for a self-similar distribution of vortices at all scales [29-31]), showing how 2D turbulent mixing layers lie between these two end-member states. When a finite $L_{d}$ is introduced, the spectra become steeper. Mixing-layer coherent structures have a more limited dynamical reach as $L_{d}$ decreases, which hinders the merging process. Rarer vortex-vortex mergers strongly decrease the filaments production around the vortices. The consequent absence of filaments obstructs the enstrophy cascade steepening and thus the spectra.

Our work promotes a mechanistic resonance-based perspective toward understanding how the behavior of vorticity waves changes with the Rossby radius of deformation. This study examines QG shear instability through the KW perspective lens. Expanding to other geophysical or astrophysical flow systems, a similar study can be conducted to evaluate the influence of $L_{d}$ on interacting gravity waves [16] or Alfvèn waves [17]. It would be interesting to observe if and to what degree these wave interactions become weakened by decreasing $L_{d}$, similarly to what occurs for CRWs.

Moreover, the introduction of a finite Rossby radius of deformation can be seen as a reasonable and intuitively useful model framework toward a better understanding of three-dimensional effects from the KW perspective. From our study of the QG model, the third dimension may be simply seen as a direction into which the kinetic energy of CRWs may be stored as potential energy in the form of fluid thickness variations. The richness of the possible modes of action are thus already apparent from our analysis of the QG model and their interpretations. Many new types of instabilities are 
known to become manifest with the introduction of the (spanwise) direction, i.e., normal to the flow. It would be intriguing to adapt the KW perspective to capture three-dimensional instabilities in more realistic and accurate models. This kind of study could have impact toward rationalizing not only geophysical flows but also more applied flow systems connected to industrial and technological applications [18,35].

\section{APPENDIX A: NUMERICAL METHOD}

The set of equations (14) and (15) is solved using standard pseudospectral methods on a doubly periodic domain. An example of the output of the code used here can be found in the chapter on turbulence describing 2D decaying processes in Ref. [8]. This means that at a given time step $t_{n}$ the $\mathrm{PV}$ is represented as

$$
q\left(x, y, t_{n}\right)=\sum_{\ell=0}^{N_{y}} \sum_{k=-N_{x}}^{N_{x}} q_{\ell, k}^{n} \exp \left[\frac{2 \pi i k x}{L_{x}}+\frac{2 \pi i \ell y}{L_{y}}\right]+\text { c.c. }
$$

in which $\ell, k, N_{x}$, and $N_{y}$ are integers. The physical size of the domain in the $x$ and $y$ directions, respectively, are $L_{x}$ and $L_{y}$. In addition, $q_{\ell, k}^{(n)}$ is the amplitude of the Fourier component at time step $n$, as indicated by a superscript. All derivative operations are assessed in Fourier space, while the nonlinear advection terms are assessed in physical space using a standard $2 / 3$ dealiasing rule. The stream function is the solution of the corresponding Poisson equation found in Eq. (15). In spectral space this becomes a simple algebraic relationship. Thus, the Fourier component of the stream function is given by

$$
\psi_{\ell, k}^{n}=-\frac{q_{\ell, k}^{(n)}}{K_{k, \ell}^{2}+L_{d}^{-2}},
$$

where the total wave number $K_{k, \ell}$ is defined as

$$
K_{k, \ell}^{2} \equiv \frac{4 \pi^{2} k^{2}}{L_{x}^{2}}+\frac{4 \pi^{2} \ell^{2}}{L_{y}^{2}} .
$$

The velocity fields, and all other derivatives, are assessed in spectral space via simple multiplication, e.g.,

$$
u_{\ell, k}^{n}=-\frac{2 \pi i \ell}{L_{y}} \psi_{\ell, k}^{(n)}, \quad v_{\ell, k}^{n}=\frac{2 \pi i k}{L_{x}} \psi_{\ell, k}^{(n)} .
$$

The PV is evolved in Fourier space. The hyperviscosity is applied during the time step scheme based on the modified Crank-Nicolson method described in Refs. [39,40]. According to this procedure, the time stepping routine then predicts the updated PV amplitude at time step $n+1$ via

$$
q_{\ell, k}^{n+1}=e^{-2 \delta t \nu_{8}\left|K_{k, \ell}\right|^{8}} q_{\ell, k}^{n-1}+2 \delta t e^{-\delta t \nu_{8}\left|K_{k, \ell}\right|^{8}} \mathcal{N}_{\ell, k}^{n},
$$

where $\mathcal{N}_{\ell, k}^{n}$ is the aforementioned nonlinear advection term in Fourier space. Most simulations were run for $N_{x}=1120$ and $N_{y}=1280$. With $2 / 3$ dealiasing rule this means there were $2 \times 800 \times 800 \approx$ $1.3 \times 10^{6}$ active Fourier modes being evolved. In typical runs we adopted values of $L_{x}=49.2928$ and $L_{y}=60$, which amounts to a typical grid spacing of about $\Delta x \approx 0.04$ and $\Delta y \approx 0.05$. For values of $L_{d}$ that were less than 10 , we considered $L_{y}=30$ instead. This adjustment was chosen since small values of $L_{d}$ means that individual vortex structures have much more limited physical reach. Also this choice was motivated by the practical observation wherein we find that runs involving small values of $L_{d}$ rarely spread in the $y$ direction. In these smaller $L_{d}$ runs we continued to keep the same number of Fourier modes in the $y$ direction. The choice of $\delta t$ typically was either 0.005 or 0.0025 . In some rare instances, when $|m|=0.5$, we found that we had to reduce the step size to as little as $\delta t=0.001$ in order to avoid violating Courant-Friedrichs-Lewy constraints. 
We usually choose values of $\nu_{8}$ in the vicinity of $1 /\left(256 \times 10^{11}\right)$. This means that for the highest resolved Fourier mode (with, say, $\delta t=0.0025$ with $k=800$ and $\ell=800$ ) the hyperviscosity reduces the amplitude by shaving off about $0.65 \%$ of the amplitude of the mode since $\exp \left(-\delta t v_{8} \mid K_{k,\left.\ell\right|^{8}}\right)=0.9935$. As a consequence, via the process of hyperviscosity alone, the highest resolved wave mode has an $e$-folding timescale roughly of about $\Delta t=0.5$. Raising the value of $v_{8}$ has visibly noticeable effects: Higher values enhance an effective stickiness between well formed vortices and tends to cause mergers to happen more readily than otherwise. Too small a value of $v_{8}$ usually results in the pileup of enstrophy and energy at the cutoff wave numbers (corresponding to that selected by $2 / 3$ dealiasing rule); in those cases the numerical experiments blow up via the generation of $2 \Delta x$ waves. We therefore settled upon the value of $v_{8}$ where we found that the observed inertial spectrum showed little to no change and the numerical experiments were free of $2 \Delta x$ instabilities.

\section{APPENDIX B: NUMERICAL VALIDATION}

We validate the behavior of the simulations by assessing the growing phase in the experiments against the linear theory predictions of Sec. III. For given values of $m$ we initiate simulations with the PV fields described above by correctly setting up the PV field with the numerically equivalent value of the background forcing $\tilde{m}$. To this PV field we add an additional small amplitude $(\sim 0.001)$ perturbation corresponding to the fastest growing mode determined from linear theory. This entails sinusoidally perturbing the two boundaries of the filament while making sure that the wavelength of the maximally growing mode $k_{\max }$ and the difference in the phase of the two waves correspond to those predicted for the maximally growing mode, i.e., $\Delta \epsilon=\Delta \epsilon_{\max }$, where $\Delta \epsilon_{\max }=\Delta \epsilon_{\max }\left(m, L_{d}\right)$ is determined from the analysis in Sec. III. The streamwise domain size of the numerical experiment is chosen to fit exactly one maximally growing mode, i.e., $L_{x}=2 \pi / k_{\max }$. The linear theory predicts a growth rate for this mode given by $\omega_{i}($ lin). We turn off hyperviscosity in its entirety and run the simulation until the profile transitions into the nonlinear regime, whereupon we end the numerical experiment. In the temporal window in which the disturbances are clearly in the linear range, we extract the perturbation kinetic energy field from the solutions and calculate an average perturbation kinetic energy $K$ (see Fig. 17). During the linear growth phase, this kinetic energy grows by four to
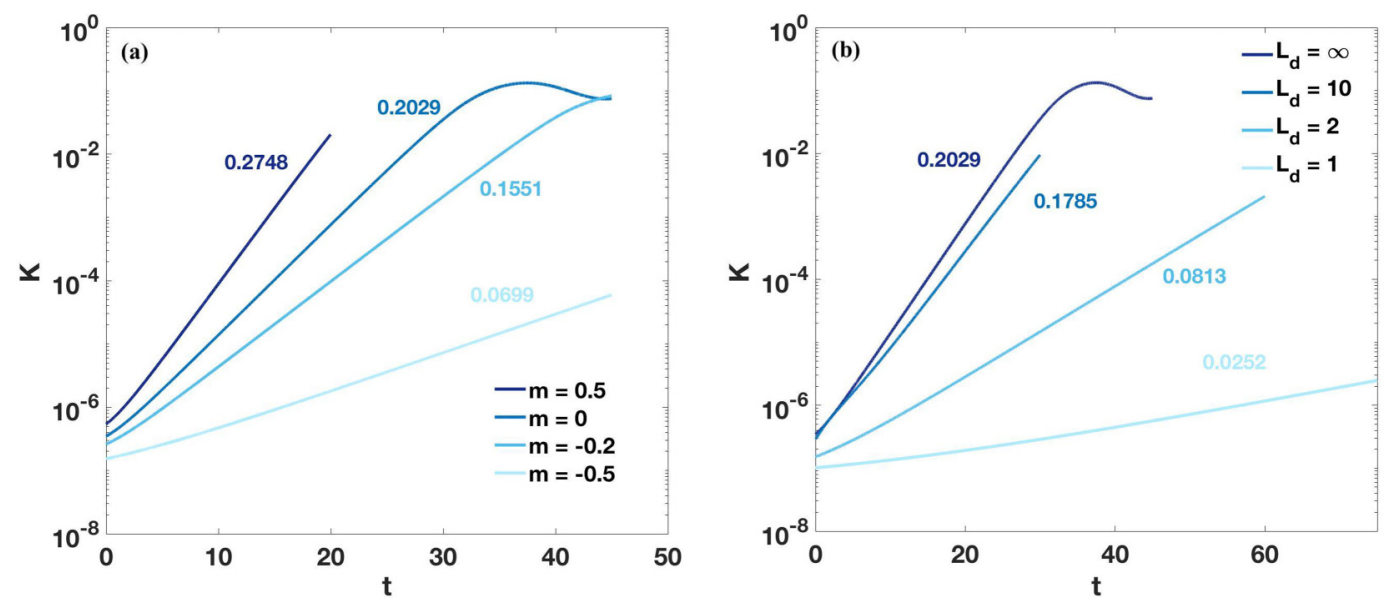

FIG. 17. Temporal behavior of the spatially averaged perturbation kinetic energy $K$ for (a) $L_{d}=\infty$ and different values of $m$ and (b) $m=0$ and different values of $L_{d}$. Note that the domain sizes are $L_{x}=\frac{2 \pi}{k_{\max }}$ (see Table I for the values of $k_{\max }$ ) and the domain height is $L_{y}=60$ for all the configurations but for $L_{d} \leqslant 2$ in which $L_{y}=30$. 
TABLE I. Summary of the test cases run for validating the nonlinear simulations and demonstrating the robustness of the equivalence conjecture.

\begin{tabular}{lccccccc}
\hline \hline$m$ & $L_{d}$ & $\tilde{m}$ & $k_{\max }$ & $\Delta \epsilon_{\max } / \pi$ & $\omega_{i}($ lin $)$ & $\omega_{i}($ DNS $)$ & Error (\%) \\
\hline 0.5 & $\infty$ & 0.500 & 0.252 & 0.6321 & 0.2763 & 0.2748 & 0.53 \\
0 & $\infty$ & 0 & 0.398 & 0.6492 & 0.2012 & 0.2029 & 0.8 \\
-0.2 & $\infty$ & -0.200 & 0.532 & 0.6419 & 0.1557 & 0.1551 & 0.38 \\
-0.5 & $\infty$ & -0.500 & 0.956 & 0.5961 & 0.0705 & 0.0699 & 0.85 \\
0.5 & 10 & 0.453 & 0.285 & 0.6060 & 0.2437 & 0.2448 & 0.45 \\
0 & 10 & 0 & 0.448 & 0.6382 & 0.1768 & 0.1785 & 0.95 \\
-0.5 & 10 & -0.453 & 1.065 & 0.5834 & 0.0566 & 0.0571 & 0.88 \\
0.5 & 2 & 0.316 & 0.302 & 0.3874 & 0.0754 & 0.0763 & 1.19 \\
0 & 2 & 0 & 0.573 & 0.5565 & 0.0810 & 0.0813 & 0.37 \\
-0.5 & 2 & -0.316 & 1.491 & 0.5439 & 0.0202 & 0.0202 & 0 \\
0 & 1 & 0 & 0.592 & 0.4843 & 0.0249 & 0.0252 & 1.20 \\
\hline \hline
\end{tabular}

five orders of magnitude before saturating. From this time series we do a linear regression fit and extract a growth rate which we call $\omega_{i}(\mathrm{DNS})$.

In Table I we summarize the results of these validations. We find that the predicted and measured values of $\omega_{i}$ differ from one another by less than $1 \%$ for all values except two simulation runs (i) $m=0.5$ and $L_{d}=2$ and (ii) $m=0$ and $L_{d}=1$, which showed errors around $1.2 \%$. Given the good correspondence between the predicted growth rates and that observed in the numerical experiments, we are confident that the numerical simulations are good and further that the equivalence conjecture we have posed is a robust concept.

[1] R. H. Kraichnan, Inertial ranges in two-dimensional turbulence, Phys. Fluids 10, 1417 (1967).

[2] R. H. Kraichnan and D. Montgomery, Two-dimensional turbulence, Rep. Prog. Phys. 43, 547 (1980).

[3] D. Bernard, G. Boffetta, A. Celani, and G. Falkovich, Conformal invariance in two-dimensional turbulence, Nat. Phys. 2, 124 (2006).

[4] G. Boffetta and R. E. Ecke, Two-dimensional turbulence, Annu. Rev. Fluid Mech. 44, 427 (2012).

[5] D. G. Dritschel, On the stabilization of a two-dimensional vortex strip by adverse shear, J. Fluid Mech. 206, 193 (1989).

[6] J. W. S. Rayleigh, The Theory of Sound (Dover, New York, 1945).

[7] P. G. Drazin, Introduction to Hydrodynamic Stability (Cambridge University Press, Cambridge, 2012).

[8] O. Regev, O. M. Umurhan, and P. A. Yecko, Modern Fluid Dynamics for Physics and Astrophysics (Springer, Berlin, 2016).

[9] F. P. Bretherton, Baroclinic instability and the short wavelength cut-off in terms of potential vorticity, Q. J. R. Meteorol. Soc. 92, 335 (1966).

[10] B. J. Hoskins, M. E. McIntyre, and A. W. Robertson, On the use and significance of isentropic potential vorticity maps, Q. J. R. Meteorol. Soc. 111, 877 (1985).

[11] E. Heifetz, C. H. Bishop, and P. Alpert, Counter-propagating Rossby waves in the barotropic Rayleigh model of shear instability, Q. J. R. Meteorol. Soc. 125, 2835 (1999).

[12] J. R. Carpenter, E. W. Tedford, E. Heifetz, and G. A. Lawrence, Instability in stratified shear flow: Review of a physical interpretation based on interacting waves, Appl. Mech. Rev. 64, 060801 (2013).

[13] P. G. Baines and H. Mitsudera, On the mechanism of shear flow instabilities, J. Fluid Mech. 276, 327 (1994).

[14] N. Harnik, E. Heifetz, O. M. Umurhan, and F. Lott, A buoyancy-vorticity wave interaction approach to stratified shear flow, J. Atmos. Sci. 65, 2615 (2008). 
[15] S. Sakai, Rossby-Kelvin instability: A new type of ageostrophic instability caused by a resonance between Rossby waves and gravity waves, J. Fluid Mech. 202, 149 (1989).

[16] A. Rabinovich, O. M. Umurhan, N. Harnik, F. Lott, and E. Heifetz, Vorticity inversion and action-at-adistance instability in stably stratified shear flow, J. Fluid Mech. 670, 301 (2011).

[17] E. Heifetz, J. Mak, J. Nycander, and O. M. Umurhan, Interacting vorticity waves as an instability mechanism for magnetohydrodynamic shear instabilities, J. Fluid Mech. 767, 199 (2015).

[18] L. Biancofiore, F. Gallaire, and E. Heifetz, Interaction between counterpropagating Rossby waves and capillary waves in planar shear flows, Phys. Fluids 27, 044104 (2015).

[19] L. Biancofiore, E. Heifetz, J. Hoepffner, and F. Gallaire, Understanding the destabilizing role for surface tension in planar shear flows in terms of wave interaction, Phys. Rev. Fluids 2, 103901 (2017).

[20] O. M. Umurhan and E. Heifetz, Holmboe modes revisited, Phys. Fluids 19, 064102 (2007).

[21] H. C. Davies and C. H. Bishop, Eady edge waves and rapid development, J. Atmos. Sci. 51, 1930 (1994).

[22] E. Heifetz, J. Methven, B. J. Hoskins, and C. H. Bishop, The counter-propagating Rossby-wave perspective on baroclinic instability. II: Application to the Charney model, Q. J. R. Meteorol. Soc. 130, 233 (2004).

[23] R. Yellin-Bergovoy, E. Heifetz, and O. M. Umurhan, Physical mechanism of centrifugal-gravity wave resonant instability in azimuthally symmetric swirling flows, Phys. Rev. Fluids 2, 104801 (2017).

[24] G. K. Vallis, Atmospheric and Oceanic Fluid Dynamics (Cambridge University Press, Cambridge, 2017).

[25] D. W. Waugh and D. G. Dritschel, The stability of filamentary vorticity in two-dimensional geophysical vortex-dynamics models, J. Fluid Mech. 231, 575 (1991).

[26] J. Liu and T. Schneider, Scaling of off-equatorial jets in giant planet atmospheres, J. Atmos. Sci. 72, 389 (2015).

[27] A. D. Gilbert, Spiral structures and spectra in two-dimensional turbulence, J. Fluid Mech. 193, 475 (1988).

[28] M. Lesieur, C. Staquet, P. Le Roy, and P. Comte, The mixing layer and its coherence examined from the point of view of two-dimensional turbulence, J. Fluid Mech. 192, 511 (1988).

[29] P. Santangelo, R. Benzi, and B. Legras, The generation of vortices in high-resolution, two-dimensional decaying turbulence and the influence of initial conditions on the breaking of self-similarity, Phys. Fluids A 1, 1027 (1989).

[30] D. G. Dritschel, A general theory for two-dimensional vortex interactions, J. Fluid Mech. 293, 269 (1995).

[31] D. G. Dritschel, R. K. Scott, C. Macaskill, G. A. Gottwald, and C. V. Tran, Unifying Scaling Theory for Vortex Dynamics in Two-Dimensional Turbulence, Phys. Rev. Lett. 101, 094501 (2008).

[32] B. H. Burgess, R. K. Scott, and T. G. Shepherd, Kraichnan-Leith-Batchelor similarity theory and twodimensional inverse cascades, J. Fluid Mech. 767, 467 (2015).

[33] E. Heifetz and J. Methven, Relating optimal growth to counterpropagating Rossby waves in shear instability, Phys. Fluids 17, 064107 (2005).

[34] Y.-Y. Hayashi and W. R. Young, Stable and unstable shear modes of rotating parallel flows in shallow water, J. Fluid Mech. 184, 477 (1987).

[35] L. Biancofiore and F. Gallaire, Counterpropagating Rossby waves in confined plane wakes, Phys. Fluids 24, 074102 (2012).

[36] C. D. Winant and F. K. Browand, Vortex pairing: The mechanism of turbulent mixing-layer growth at moderate Reynolds number, J. Fluid Mech. 63, 237 (1974).

[37] C.-M. Ho and P. Huerre, Perturbed free shear layers, Annu. Rev. Fluid Mech. 16, 365 (1984).

[38] L. Biancofiore, Crossover between two-and three-dimensional turbulence in spatial mixing layers, J. Fluid Mech. 745, 164 (2014).

[39] O. M. Umurhan and O. Regev, Hydrodynamic stability of rotationally supported flows: Linear and nonlinear 2D shearing box results, Astron. Astrophys. 427, 855 (2004).

[40] T. Tamarin, E. Heifetz, O. M. Umurhan, and R. Yellin, On the nonnormal-nonlinear interaction mechanism between counter-propagating Rossby waves, Theor. Comput. Fluid Dyn. 29, 205 (2015).

[41] C. Basdevant, B. Legras, R. Sadourny, and M. Beland, A study of barotropic model flows: Intermittency, waves and predictability, J. Atmos. Sci. 38, 2305 (1981).

[42] J. C. McWilliams, The emergence of isolated coherent vortices in turbulent flow, J. Fluid Mech. 146, 21 (1984). 
[43] B. Legras, P. Santangelo, and R. Benzi, High-resolution numerical experiments for forced twodimensional turbulence, Europhys. Lett. 5, 37 (1988).

[44] J. Weiss, The dynamics of enstrophy transfer in two-dimensional hydrodynamics, Physica D 48, 273 (1991).

[45] G. Boffetta and S. Musacchio, Evidence for the double cascade scenario in two-dimensional turbulence, Phys. Rev. E 82, 016307 (2010).

[46] P. Tabeling, Two-dimensional turbulence: A physicist approach, Phys. Rep. 362, 1 (2002). 